\title{
One-loop Electroweak and QCD corrections to vector boson scattering into top pairs and application to the ILC
}

\author{
N. Bouayed ${ }^{1,2)}$ and F. Boudjema ${ }^{2)}$ \\ 1) Université Sâad Dahlab de Blida, route de Soumâa, B.P.270, 09000 Blida, Algeria \\ 2) $\mathrm{LAPTH}^{\dagger}$, Université de Savoie, CNRS, 9 chemin de Bellevue, B.P.110, \\ F-74941 Annecy-le-Vieux, France.
}

\begin{abstract}
We calculate the electroweak and QCD corrections to $W^{-} W^{+} \rightarrow t \bar{t}$ and $Z Z \rightarrow t \bar{t}$. We also consider the interplay of these corrections with the effect of anomalous interactions that affect the massive weak bosons and the top. The results at the $V V$ level fusion are convoluted with the help of the effective vector boson approximation to give predictions for a high energy $e^{+} e^{-}$collider.
\end{abstract}

†Laboratoire d'Annecy-le-Vieux de Physique Théorique, UMR 5108. 


\section{Introduction}

The fact that the Higgs has not been discovered yet is evidence that the mechanism behind electroweak symmetry breaking, EWSB, giving masses not only to the weak vector bosons but also to fermions remains a mystery. Moreover the implementation of the Higgs within the Standard Model, $\mathcal{S} \mathcal{M}$, poses a few conceptual problems. This has triggered an intense activity in model building in order to solve the hierarchy problem leading to a rich phenomenology at the upcoming high energy colliders, such as the Large Hadron Collider (LHC) [1] and the International Linear Collider (ILC) [2]

In practically all the New Physics models which address the hierarchy problem the top plays a central and crucial role. One can mention models of dynamical electroweak symmetry breaking in the technicolour vein where the concept of top condensation [3] is exploited. In supersymmetry, the top keeps the the so-called minimal model alive through radiative corrections to the Higgs while in mSUGRA, for example, the top can trigger electroweak symmetry breaking. The phenomenology of the top in models of extra-dimensions is also special as discussed very recently [4]. It is no wonder that the top plays such a central role in probing the sector of EWSB as well as the flavour problem. After all, its large mass [5] is of the order of the spontaneous electroweak symmetry scale $v / \sqrt{2}=174 \mathrm{GeV}$ which, in the $\mathcal{S M}$, gives it a Yukawa coupling of order one, incidentally sensibly of the same strength as the QCD coupling constant at the EWSB scale. This also explains why radiative corrections involving the top, especially in processes related to symmetry breaking, may be competitive with QCD corrections. It is in this context that we compute in this paper the electroweak corrections to vector boson fusion into a pair of top quarks, $Z Z \rightarrow t \bar{t}$ and $W^{-} W^{+} \rightarrow t \bar{t}$. Weak vector boson fusion is the privileged process revealing spontaneous symmetry breaking through the longitudinal or Goldstone component of the vector bosons. Although most analyses have dealt with scattering into vector bosons, it is clear that fusion into tops is also a probe of symmetry breaking. Since the effects of the EWSB due to New Physics can be subtle, it is important that we know quite precisely the $\mathcal{S M}$ prediction. This calls for calculations beyond tree-level. QCD corrections have been performed recently in [6]. We will include here the electroweak corrections alongside the QCD corrections. We also compare the loop corrections effects with those from new operators parameterising the EWSB effects in $W^{-} W^{+} \rightarrow t \bar{t}$. The complete study of vector boson fusion at the colliders would require a full $2 \rightarrow 4$ calculation. Especially for the inclusion of radiative corrections this is a most daunting task. Though most of the machinery has been developed to tackle such complex $2 \rightarrow 4$ processes at loop level, there have been till now only two such computations [7, 8]. It therefore seems fit in a first step to revert to some approximation. The effective vector boson approximation, EVBA [9], seems suited for such processes. This approximation has been extensively used in the context of weak vector fusion. We will conduct our calculation within this approximation for application to the $e^{+} e^{-}$linear collider. The important genuine electroweak corrections should be captured in the subprocess. We only restrict ourselves to the linear collider; $W^{-} W^{+} \rightarrow t \bar{t}$ in the context of the LHC suffers from considerable background[10] mainly due to the overwhelming direct $t \bar{t}$ production. The Higgs range we consider in this paper does not go beyond a Higgs mass, $M_{\text {Higgs }}$, of $300 \mathrm{GeV}$ in accordance with the current limit from precision measurements [11]. We intend to study the radiative corrections in the presence of a very heavy Higgs in a forthcoming publication.

This paper is organized as follows. In the next section we briefly review $Z Z \rightarrow t \bar{t}$ and $W^{-} W^{+} \rightarrow t \bar{t}$ and show the importance of the longitudinal modes especially at high energies. We then expose the EVBA. In section 3 we present the main ingredients that enter the calculations at one-loop, the checks we performed as well as the tools we exploited. Section 4 gives our results for the electroweak and QCD corrections. Section 5 shows these results after convolution with the EVBA structure functions for the linear collider. Section 6 introduces the anomalous operators and their effects on the processes we study. Section 7 summarises our conclusions. An appendix lists all formulae for the helicity amplitudes at tree-level, in a compact way, including the anomalous operators. 


\section{Tree-level overview and dominance of the longitudinal modes}

\subsection{Notation and Born contribution}

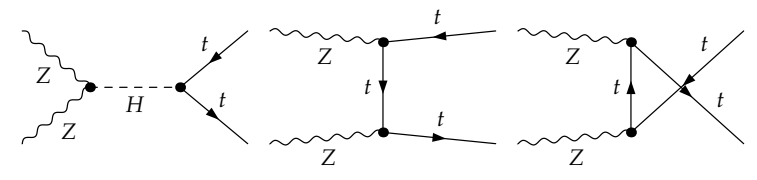

(a) $Z Z \rightarrow t \bar{t}$

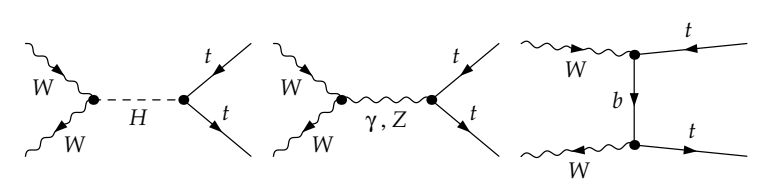

(b) $W^{-} W^{+} \rightarrow t \bar{t}$

FIG. 1: Tree level Feynman diagrams for the process a) $Z Z \rightarrow \bar{t} t$ and b) $W^{-} W^{+} \rightarrow \bar{t} t$.

The tree level Feynman diagrams for the processes we study, $Z\left(k_{1}, \lambda_{1}\right)+Z\left(k_{2}, \lambda_{2}\right) \rightarrow t\left(k_{3}, ; \lambda_{3}\right)+$ $\bar{t}\left(k_{4}, \lambda_{4}\right)$ and $W^{-}\left(k_{1}, \lambda_{1}\right)+W^{+}\left(k_{2}, \lambda_{2}\right) \rightarrow t\left(k_{3}, ; \lambda_{3}\right)+\bar{t}\left(k_{4}, \lambda_{4}\right)$ are shown in figure 1(a) and 1(b). The labelling $k_{i}$ and $\lambda_{i}= \pm, 0$ stand for the momentum and the helicity. Complete helicity amplitudes formulae for $W^{-} W^{+} \rightarrow t \bar{t}$ are given in the Appendix.

We are mostly interested in the longitudinal modes of the vector bosons, with $\lambda_{1}=\lambda_{2}=0 \equiv L$. Total cross sections for this mode are shown in Figs 2 ,

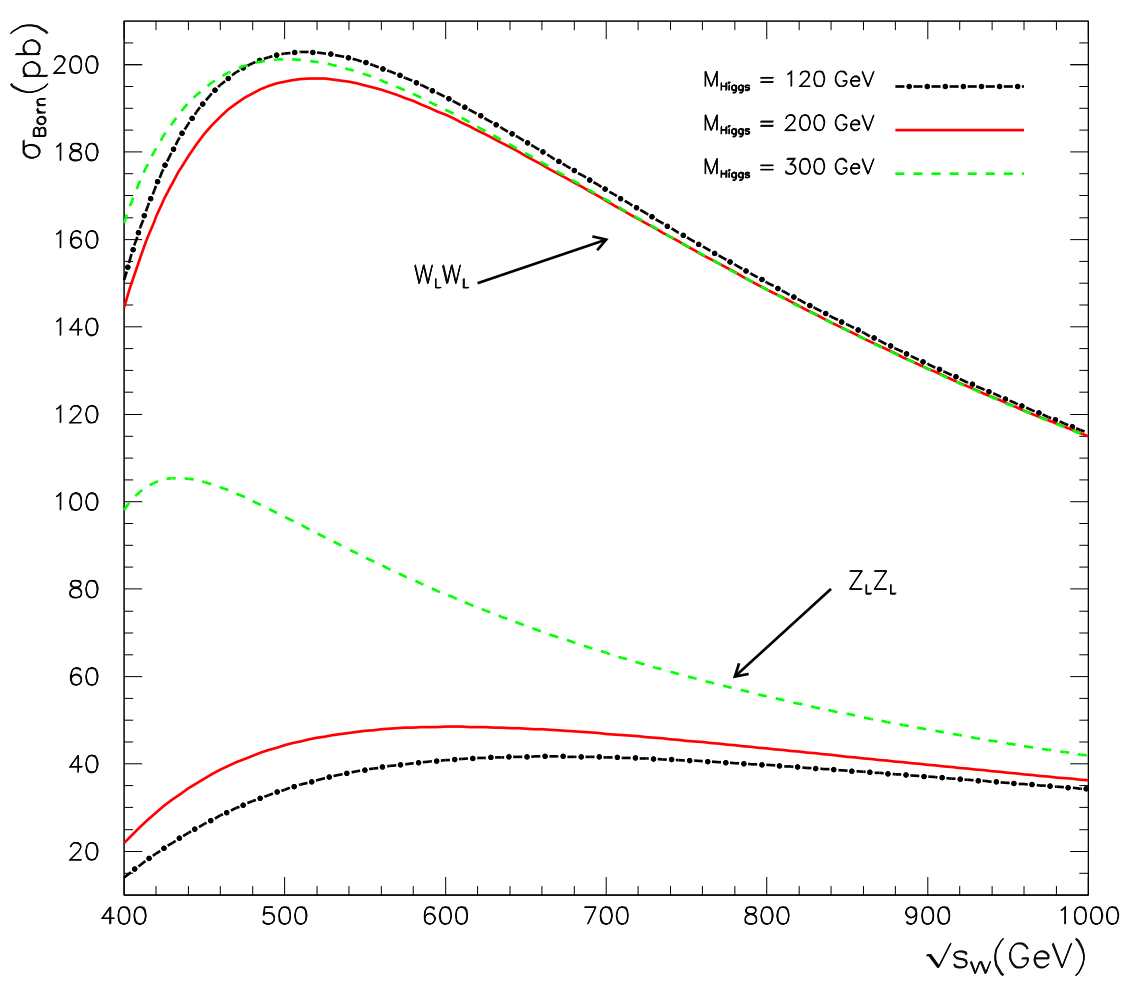

FIG. 2: Total Born cross section for the processes $W_{L}^{-} W_{L}^{+} \rightarrow \bar{t} t$ and $Z_{L} Z_{L} \rightarrow \bar{t} t$ for $M_{\text {Higgs }}=$ $120,200,300 \mathrm{GeV}$ and $p_{T}^{t, \bar{t}}>10 \mathrm{GeV}$.

$Z_{L} Z_{L}$ is more sensitive to the Higgs mass dependence especially for low centre of mass energies. The $W$ mode provides though more yield. Note also, that for the range of Higgs masses we are studying the cross sections after reaching a maximum around threshold, drop as the energy increases. This should be taken into account when considering the effect of New Physics (NP) that tends to increase the cross section as the energy increases. When convoluting with the "structure function" of the EVBA (see next), the lower end of the cross section might pollute the NP effects. One should therefore aim at imposing a cut on the invariant mass of the system. We will always take a cut on the invariant mass of the $t \bar{t}$, 
$m_{t \bar{t}}>400 \mathrm{GeV}$ as is done in [6]. This cut will help improve the EVBA. We will also impose a cut on the transverse momentum of the top, $p_{T}^{t, \bar{t}}>10 \mathrm{GeV}$. We will do this not only after convolution on the structure function, i.e. at the $e^{+} e^{-}$level but also at the $V V \rightarrow t \bar{t}$ level. The figures we show are with these cuts. The $p_{T}^{t, \bar{t}}$ cut helps reduce some QCD background and fake $p_{T}$ in $t \bar{t} \gamma$, see[12, 13, 14].
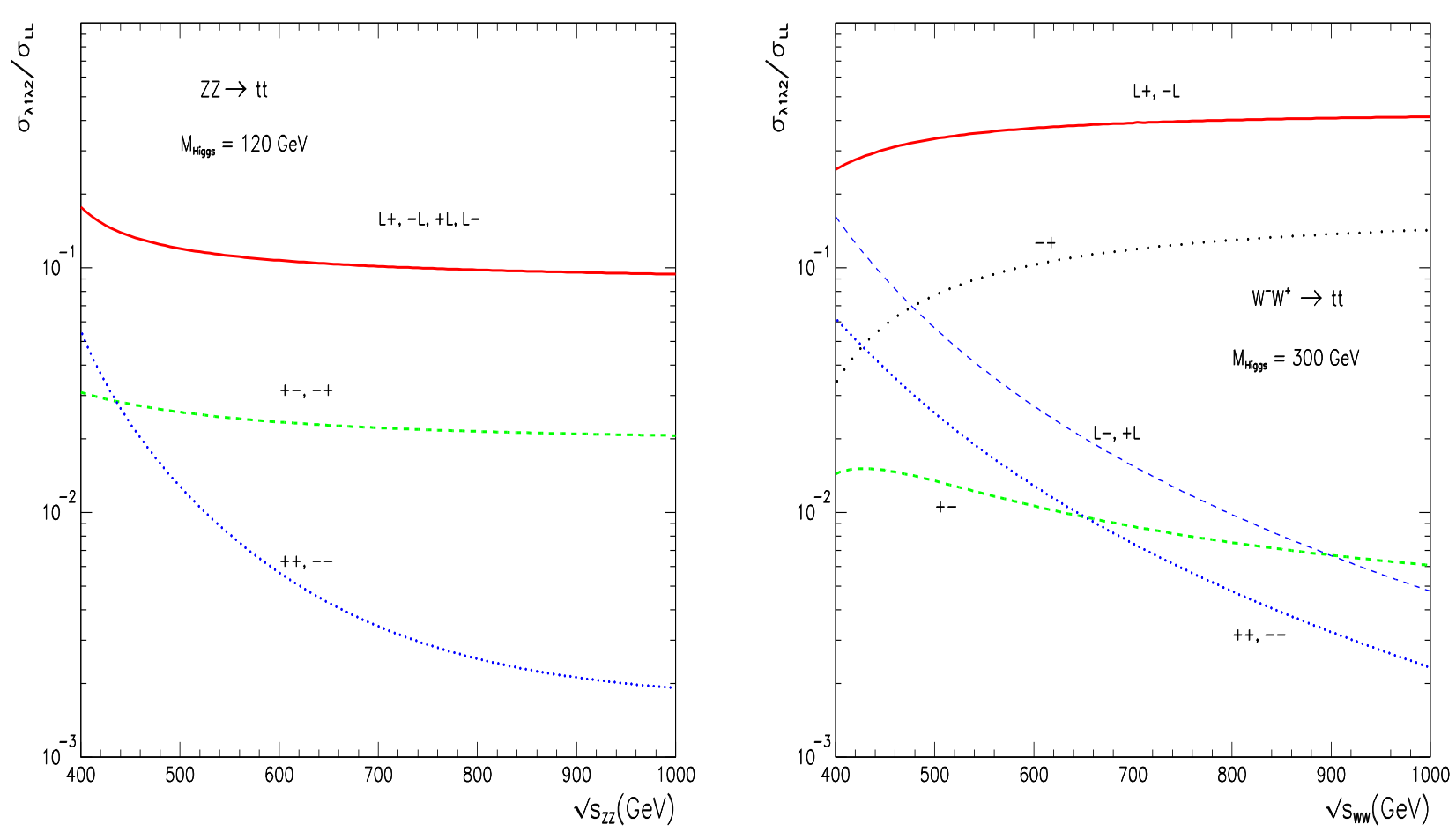

FIG. 3: Relative rates for the Born cross section for different polarisations of the incoming vector bosons with respect to the case where the incoming bosons are both longitudinally polarised. For $W W, \lambda_{i} \lambda_{j}$ labelling of the helicities refers to $W^{-}\left(\lambda_{i}\right) W^{+}\left(\lambda_{j}\right)$.

Another reason for aiming at a higher cut on the invariant $V V$ (or similarly $t \bar{t}$ ) system can be gleaned in Fig. 3. First $V_{L} V_{L}$ scattering dominates for all energies. This said, at the lowest centre of mass energies, some transverse contributions might not be so negligible as compared to $V_{L} V_{L}$ fusion, though they drop rather rapidly as the energy increases. Note however that the longitudinal/transverse $\left(V_{L} V_{T}\right)$ mode in the $\mathcal{S M}$ might bring some contamination at all energies, which could worsen the EVBA approximation.

\subsection{Born angular distributions}

Radiative corrections and the New physics can not only change the total yield but can also, and most dramatically, distort the shape of kinematical distributions. We show in Fig. 4 the angular distribution in $W^{-} W^{+} \rightarrow t \bar{t}$ for $\sqrt{s_{W W}}=400 \mathrm{GeV}$, not too far from threshold and at high energy $\sqrt{s_{W W}}=1 \mathrm{TeV}$. We choose to show $W^{-} W^{+} \rightarrow t \bar{t}$ because of the chiral structure of the $W$ coupling and the small mass of the bottom (exchanged in the $t$-channel). This leads, at high energy, to an overwhelming production of top in the backward region, with respect to $W^{-}$. In $Z Z$, the distribution are of course symmetric and much less peaked. A cut on the forward backward region will also help bring out the signal of New Physics which occurs at higher $p_{T}$. 

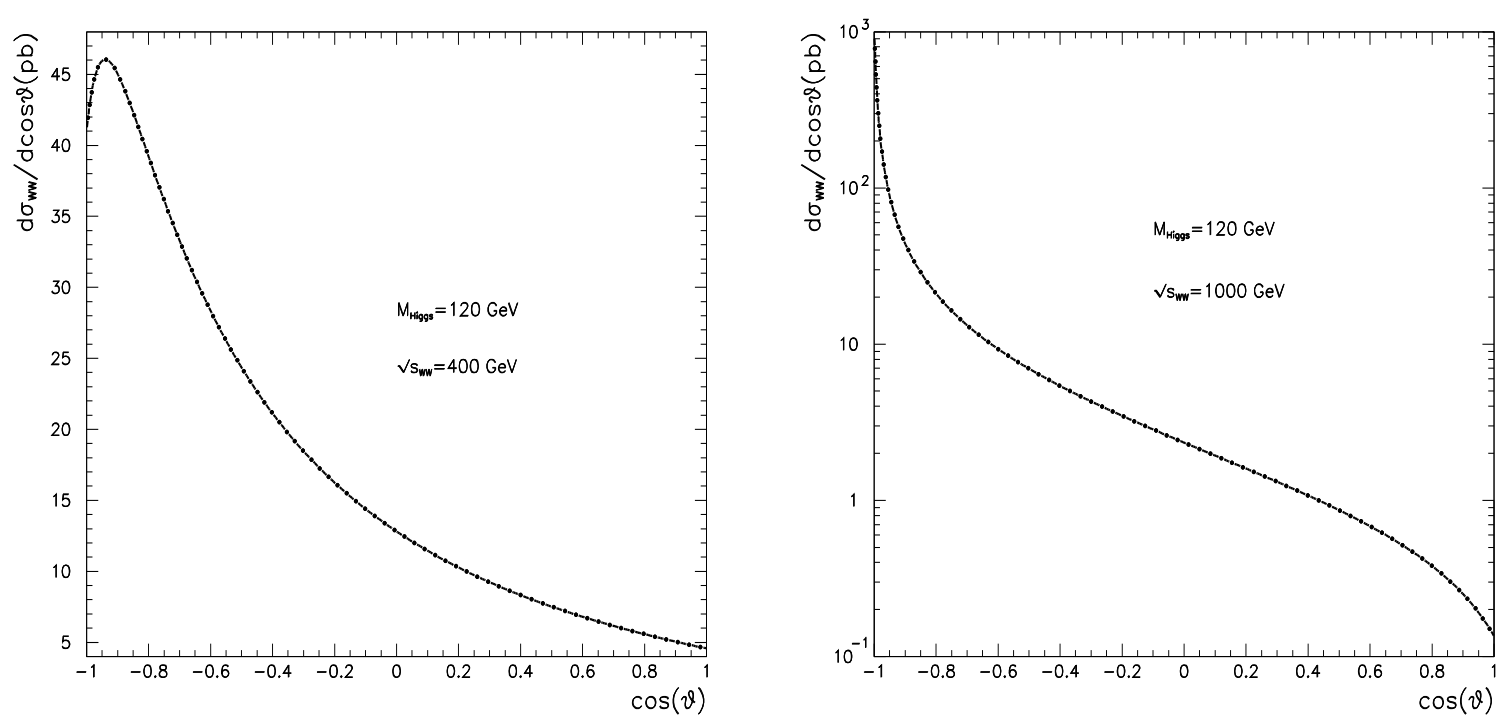

FIG. 4: Angular distribution for the unpolarised $W^{-} W^{+} \rightarrow t \bar{t}$ cross section for $M_{\text {Higgs }}=120 G e V$ at $\sqrt{s_{W W}}=400 \mathrm{GeV}$ and $1 \mathrm{TeV}$. $\theta$ is the angle between the $W^{-}$and the $t$. The $p_{T}^{t, \bar{t}}>10 \mathrm{GeV}$ corresponds to a too small $\theta_{\text {cut }}$ that can not be represented on the plot.

\subsection{Weak bosons effective distribution functions}

To turn the subprocess $Z Z \rightarrow t \bar{t}$ and $W^{-} W^{+} \rightarrow t \bar{t}$ into $e^{+} e^{-} \rightarrow t \bar{t} e^{+} e^{-}$and $e^{+} e^{-} \rightarrow t \bar{t} \nu_{e} \bar{\nu}_{e}$ respectively, we revert to the EVBA approximation[9]. The approximation introduces the notion of the $W / Z$ content of the electron (and positron). At high energies, we take the structure functions of the weak vector bosons inside a fermion as given by [15, 16] :

$$
\begin{gathered}
f_{\text {fermion } / V, \lambda}(x)=\frac{1}{16 \pi x}\left[\left(g_{V}-\lambda g_{A}\right)^{2}+(1-x)^{2}\left(g_{V}+\lambda g_{A}\right)^{2}\right]\left[\ln \frac{P_{T, \max }^{2}}{M_{V}^{2}}-1\right], \quad \lambda= \pm 1 \\
f_{\text {fermion } / V, \lambda}(x)=\frac{g_{V}^{2}+g_{A}^{2}}{4 \pi^{2}} \frac{1-x}{x}, \quad \lambda=0,
\end{gathered}
$$

$x$ is the momentum fraction transferred to the vector boson by the initial fermion. $g_{V}$ and $g_{A}$ are the vector and axial-vector couplings of the weak vector bosons to fermions. The transverse distribution is subject to more uncertainty due to the logarithmic factor. In our case, we take $P_{T, \max }$ the maximum transverse momentum of the vector bosons allowed by the kinematics. In the collision of two elementary fermions $f_{1}$ and $f_{2}$ of respective charge and isospin assignement $\left(Q_{f_{1}}, T_{f_{1}}^{(3)}\right)$ and $\left(Q_{f_{2}}, T_{f_{2}}^{(3)}\right)$, the effective luminosity of the longitudinal vector bosons is given by:

$$
\left.\frac{d \mathcal{L}}{d \tau}\right|_{f_{1} f_{2} / V_{L} V_{L}}(\tau)=\int_{\tau}^{1} \frac{d x}{x} f_{f_{1} / V_{L}}(x) f_{f_{2} / V_{L}}\left(\frac{\tau}{x}\right) .
$$

This gives for example for $W_{L} W_{L}[17]$ :

$$
\left.\frac{d \mathcal{L}}{d \tau}\right|_{f_{1} f_{2} / W_{L} W_{L}}(\tau)=\left[\frac{\alpha}{4 \pi S_{W}^{2}}\right]^{2} \frac{1}{\tau}\left((1+\tau) \ln \frac{1}{\tau}-2(1-\tau)\right) .
$$

The total cross section for the processes $\left(e^{+} e^{-\stackrel{W_{L} W_{L}}{\longrightarrow}} t \bar{t}+\nu_{e} \bar{\nu}_{e}\right)$ is then given by:

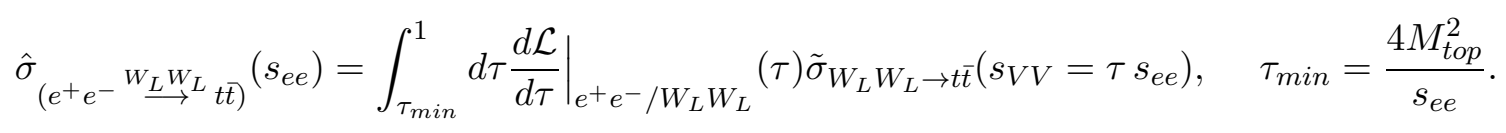


Formulae for the $Z Z$ case can be derived in a similar manner.

After convolution, the $e^{+} e^{-}$cross section to $t \bar{t}$ through vector boson fusion is reduced much and will most probably be exploited only in a second stage linear collider with energy in excess of $1 \mathrm{TeV}$, see Fig. 5. Note that at the level of $e^{+} e^{-}$, the litte Higgs mass dependence there is in the $W W$ channel $\left(M_{\text {Higgs }}=120 \mathrm{GeV}\right.$ and $\left.M_{\text {Higgs }}=300 \mathrm{GeV}\right)$ is washed out but not in the $Z Z$ channel, though the latter cross section is now, at least, an order of magnitude smaller than in $W W$ suffering from the smaller couplings of the $Z$ to the electrons.

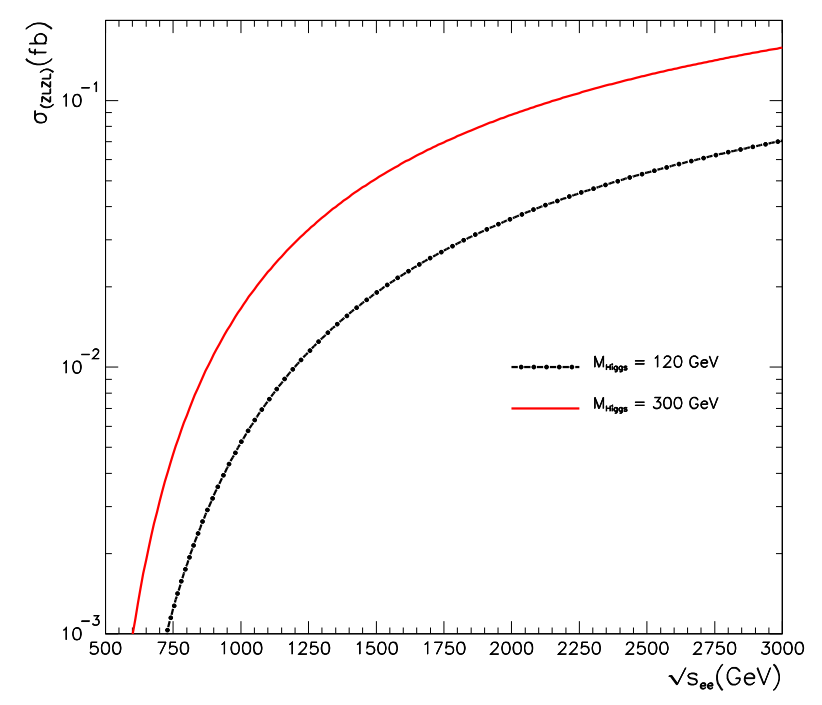

(a) $e^{+} e^{-} \rightarrow t \bar{t} e^{+} e^{-}$through $Z_{L} Z_{L} \rightarrow t \bar{t}$

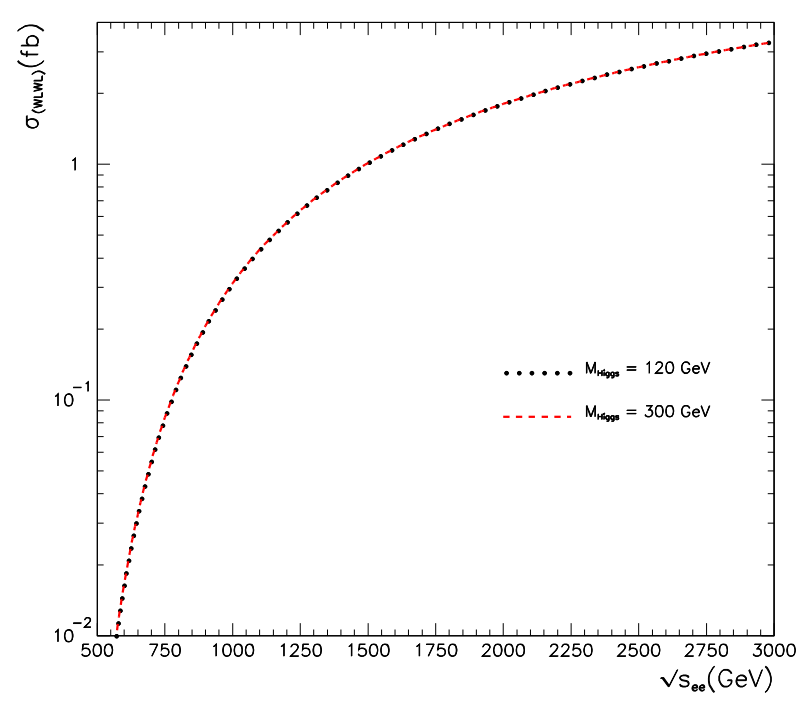

(b) $e^{+} e^{-} \rightarrow t \bar{t} \nu_{e} \bar{\nu}_{e}$ through $W_{L} W_{L} \rightarrow t \bar{t}$

FIG. 5: Born total cross sections for (a) $e^{+} e^{-} \rightarrow t \bar{t} e^{+} e^{-}$through $Z_{L} Z_{L} \rightarrow t \bar{t}$ and (b) $e^{+} e^{-} \rightarrow t \bar{t} \nu \bar{\nu}$ process through $W_{L}^{-} W_{L}^{+} \rightarrow t \bar{t}$.

One should keep in mind that the effective EVBA works best at high energy (compared to the $W$ mass for instance). Reference [13 made a comparison of the EVBA with a full SM calculation (at tree-level) using CompHEP [18]. It was found that the EVBA is a good approximation for $\sqrt{s_{e e}}=1.5 \mathrm{TeV}$ and above.

\section{Electroweak and QCD corrections: Set up and details of the calculation}

\subsection{General structure}

The calculation of the complete one-loop electroweak and QCD corrections to $W^{-} W^{+} \rightarrow t \bar{t}$ and $Z Z \rightarrow t \bar{t}$ is performed with the help of two automatic codes for loop corrections, SloopS[19] and FormCalc/LoopTools [20]. Although SloopS uses many modules of [20], the model files and the generation of the Feynman rules is generated automatically with the help of LanHep 21]. Moreover, some loop integration routines have been improved. The strength of SloopS is the ability to perform very powerful gauge parameter independence checks as we will see later, a feature that is found in GRACE-loop 22]. SloopS has been developed for supersymmetry but has also a $\mathcal{S M}$ module. The results of SloopS, FormCalc/LoopTools and GRACE-loop have been checked for a variety of electroweak processes. All codes adopt the on-shell renormalisation scheme according to[22, 23, 24]. In particular we take as input, the masses of the particles in the model and the electromagnetic coupling as defined in the Thomson limit. For the process at hand, this means $\alpha$ the electromagnetic coupling, the masses of the $W, M_{W}$, the $Z, M_{Z}$ and the top $m_{t}$. 

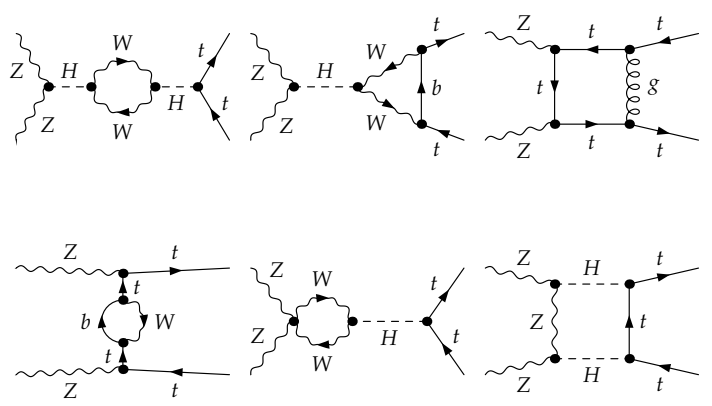

(a) $Z Z \rightarrow t \bar{t}$
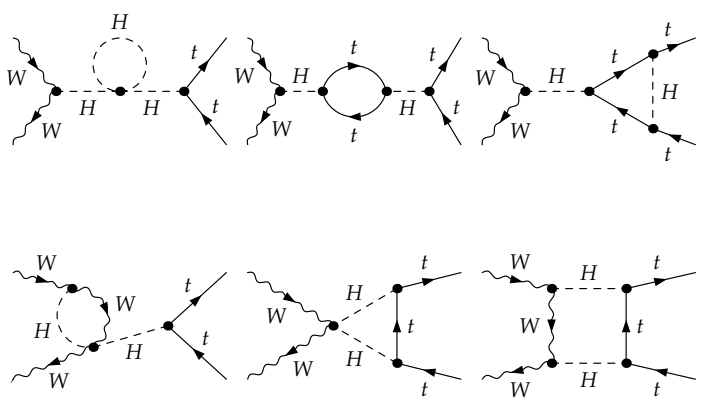

(b) $W^{-} W^{+} \rightarrow t \bar{t}$

FIG. 6: Some one loop Feynman diagrams for the processes a) $Z Z \rightarrow \bar{t} t$ and b) $W^{-} W^{+} \rightarrow \bar{t} t$.

The one-loop amplitudes consist of the virtual corrections $\mathcal{M}_{1 \text { loop }}^{E W+Q C D}$ and the counterterm contributions $\mathcal{M}_{C T}$. Fig. 6 shows a selection of the virtual corrections. There are about 500 diagrams for the process $W^{-} W^{+} \rightarrow t \bar{t}$ and 400 for the process $Z Z \rightarrow t \bar{t}$. Though $\mathcal{M}_{1 \text { loop }}^{E W+Q C D}+\mathcal{M}_{C T}$ should be ultraviolet finite, photon and gluon virtual exchange leads to infrared divergences. These are regulated by a small photon or gluon mass. For the latter the procedure is admissible as we are essentially dealing with an Abelian process where the triple gluon vertex for example does not show up. The photon and gluon mass regulator contribution contained in the virtual correction should cancel exactly against the one present in the photon and gluon final state radiation. A selection of diagrams in the latter category is listed in Fig. 7.

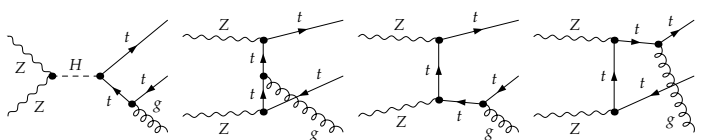

(a) $Z Z \rightarrow t \bar{t} g$

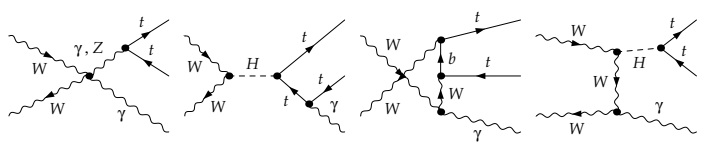

(b) $W^{-} W^{+} \rightarrow t \bar{t} \gamma$

FIG. 7: A selection of the final state radiation diagrams for the process a) $Z Z \rightarrow t \bar{t} g$ and b) $W^{-} W^{+} \rightarrow t \bar{t} \gamma$.

The photonic (gluonic) contribution is in fact split into a soft part, where the photon (gluon) energy is less than some small cut-off $k_{c}, \mathcal{M}_{\gamma, g}^{\text {soft }}\left(E_{\gamma, g}<k_{c}\right)$ and a hard part with $\mathcal{M}_{\gamma, g}^{\text {hard }}\left(E_{\gamma, g}>k_{c}\right)$. The former requires a photon/gluon mass regulator. We use the usual universal factorised form with a simple rescaling for the case of the gluon correction. To summarise, the full one-loop electroweak correction is the result of all these contributions:

$$
\left|\mathcal{M}_{1}\right|^{2, E W}=\overbrace{2 \mathcal{R} e \mathcal{M}_{\text {Born }}\left(\mathcal{M}_{1 \text { loop }}^{E W}+\mathcal{M}_{C T}\right)^{*}+\left|\mathcal{M}_{\gamma}^{\text {soft }}\right|^{2}}^{\left|\mathcal{M}_{1}\right|^{2, s+v}}+\left|\mathcal{M}_{\gamma}^{\text {hard }}\right|^{2}
$$

The QCD correction is defined along the same line. The percentage change, for the total electroweak correction including hard photon radiation will be defined as $\delta^{E W}$. For the QCD correction we will define this as $\delta^{Q C D}$.

\subsection{Checks on the calculation}

i) We first check the ultraviolet (UV) finiteness of the results. This test applies to the whole set of the virtual one-loop diagrams. The ultraviolet finiteness test is performed by varying the dimensional regularisation parameter $C_{U V}=1 / \varepsilon-\gamma_{E}+\log 4 \pi, n=4-2 \varepsilon$ is the dimensionality of space-time. We vary $C_{U V}$ by seven orders of magnitude with no change in the result. We content ourselves here and all other tests with double precision. The $C_{U V}$ parameter could then be set to 0 in further computations. 
ii) The test on the infrared (IR) finiteness is performed by including both the loop and the soft bremsstrahlung contributions and checking that there is no dependence on the fictitious photon mass $\lambda_{\gamma}$ or gluon mass $\lambda_{g}$.

iii) A crucial test concerns the gauge parameter independence of the results. Gauge parameter independence of the result is performed through a set of five gauge fixing parameters. For the latter a generalised non-linear gauge fixing condition [22, 25] has been chosen,

$$
\begin{aligned}
\mathcal{L}_{G F}= & -\frac{1}{\xi_{W}}\left|\left(\partial_{\mu}-i e \tilde{\alpha} A_{\mu}-i g c_{W} \tilde{\beta} Z_{\mu}\right) W^{\mu+}+\xi_{W} \frac{g}{2}\left(v+\tilde{\delta} H+i \tilde{\kappa} \chi_{3}\right) \chi^{+}\right|^{2} \\
& -\frac{1}{2 \xi_{Z}}\left(\partial . Z+\xi_{Z} \frac{g}{2 c_{W}}(v+\tilde{\varepsilon} H) \chi_{3}\right)^{2}-\frac{1}{2 \xi_{A}}(\partial . A)^{2}, \quad c_{W}=M_{W} / M_{Z} .
\end{aligned}
$$

The $\chi$ represents the Goldstone. We take the 't Hooft-Feynman gauge with $\xi_{W}=\xi_{Z}=\xi_{A}=1$ so that no "longitudinal" term in the gauge propagators contributes. Not only does this make the expressions much simpler and avoids unnecessary large cancellations, but it also avoids the need for high tensor structures in the loop integrals. The use of the five parameters, $\tilde{\alpha}, \tilde{\beta}, \tilde{\delta}, \tilde{\kappa}, \tilde{\varepsilon}$ is not redundant as often these parameters check complementary sets of diagrams. It is important to note that in order to successfully achieve this test one should not include any width in the propagators. In fact our tree-level results do not include any width. Because of the parameters and the energies we consider, no width is required to regularise the cross section. We verified that the inclusion of a width, at tree-level, for the Higgs is minuscule. At one-loop order, effective running widths are generated.

iv)Phase space integration for the $2 \rightarrow 2$ process is performed using the Gauss-Patterson method. While for integration connected to $2 \rightarrow 2+\gamma$ processes, we use VEGAS adaptive Monte Carlo integration package [26]. For the radiative process, we choose $k_{c}$ small enough and verify the calculation for the total unpolarised cross section with the help of CompHEP [18]. We then check the stability and independence of the result for the total and differential corrections, Eq. 3.6, with respect to $k_{c}$.

\subsection{Extraction of some photonic corrections, genuine electroweak corrections}

The QED corrections contained in the electroweak corrections may be important corrections however they do not reveal much about the inner structure. We do not expect their contribution to be large as in the case of the $e^{+} e^{-}$initiated processes which are dominated by collinear singularities. This is because of the large mass of the $W$ and top. Nonetheless one can attempt to extract their effect, assuming such a split between weak and QED is possible. For $W^{-} W^{+} \rightarrow t \bar{t}$ this is not trivial because of the non-abelian nature of the photon radiation from the $W$. This said the extraction of the leading log, $k_{c}$ dependent, contribution in the virtual+soft can be considered as a genuine QED correction. For $Z Z \rightarrow t \bar{t}$ this QED correction can be extracted most easily by a simple adaptation of the one loop initial state radiation, ISR, in $e^{+} e^{-}$. We thus take, with $Q_{t}=2 / 3$ the charge of the top, the relative QED correction to be:

$$
\delta^{q e d, s+v}=Q_{t}^{2} \frac{2 \alpha}{\pi}\left(\frac{1+\beta^{2}}{2 \beta} \ln \frac{1+\beta}{1-\beta}-1\right) \ln \frac{2 k_{c}}{\sqrt{s}}, \quad \beta=\sqrt{1-\frac{4 m^{2}}{s}} .
$$

At threshold, $\beta \rightarrow 0$, the QED corrections can be extremely large. This is due to the Coulomb correction. This can be extracted from:

$$
\begin{aligned}
\delta_{\text {Coul }}^{q e d, s+v} & =Q_{t}^{2} \frac{2 \alpha}{\pi} \frac{1+\beta^{2}}{2 \beta}\left[\operatorname{Sp}\left(\frac{2 \beta}{\beta-1}\right)-\operatorname{Sp}\left(\frac{2 \beta}{\beta+1}\right)+\frac{\pi^{2}}{2}\right], \quad S p(z)=-\int_{0}^{z} d t \frac{\log (1-t)}{t} \\
\delta_{\text {Coul }}^{q e d, s+v}(\beta \rightarrow 0) & =Q_{t}^{2} \frac{\pi \alpha}{2 \beta} .
\end{aligned}
$$

This Coulombic correction is also present in $W^{-} W^{+} \rightarrow t \bar{t}$. The corresponding QCD correction is obtained by $Q_{t}^{2} \alpha \rightarrow C_{F} \alpha_{s}$, with $C_{F}=4 / 3$ or in effect $\alpha \rightarrow 3 \alpha_{s}$. 
For $Z Z \rightarrow t \bar{t}$ the "purely" electroweak correction $(P E W)$ is defined from the contribution of the soft and virtual corrections, i.e. from $\left|\mathcal{M}_{1}\right|^{2, s+v}$ in Eq. 3.6 and therefore does not include hard photon radiation. To this we subtract the QED correction given by Eq. 3.8 so that:

$$
\sigma_{Z Z}^{P E W}=\sigma_{Z Z}^{s+v}-\delta^{q e d, s+v} \sigma_{Z Z}^{B o r n}=\delta^{P E W} \sigma_{Z Z}^{\text {Born }} .
$$

This expression is gauge invariant, UV and IR finite and independent of the photon energy cutoff.

For $W^{-} W^{+} \rightarrow t \bar{t}, \delta^{q e d, s+v}$ involves radiation from the initial, the final as well as their interference. Since we are only seeking the $k_{c}$ dependent term, we decided to extract it numerically. To achieve this we take 2 different values of the cut $k_{c}$, say $k_{c 1}$ and $k_{c 2}$. Then, for the integrated cross section the purely electroweak correction is defined as:

$$
\sigma_{W W}^{P E W}=\sigma_{W W}^{s+v}\left(k_{c}\right)-A(\sqrt{s}) \ln \left(2 k_{c} / \sqrt{s}\right), \quad A(\sqrt{s})=\frac{\sigma_{W W}^{E W}\left(k_{c 2}\right)-\sigma_{W W}^{E W}\left(k_{c 1}\right)}{\ln \left(k_{c 2} / k_{c 1}\right)}
$$

\subsection{Genuine electroweak corrections in the $G_{\mu}$ scheme}

As stated earlier we work in a scheme where the electromagnetic coupling, $\alpha$, is defined in the Thomson limit while our processes occur at the $\mathrm{TeV}$ scale. The running $\alpha$ can induce large corrections due essentially to the light fermions. Moreover some $m_{t}^{2}$ universal correction from $\Delta \rho$ can also be induced. It is therefore more advantageous to work in the $G_{\mu}$ scheme. This helps extract the rather large universal corrections contained in the two point function of the vector bosons self-energies through $\Delta r$ which is of order $3 \%$ for the Higgs masses we are considering. In this scheme the electroweak corrections are obtained by subtracting $2 \Delta r$ from the percentage change for the $P E W$ correction $\delta^{P E W}$, hence defining $\delta^{\text {pew }}=\delta^{P E W}-2 \Delta r$.

\section{One loop electroweak and QCD corrections: results}

\subsection{Input parameters}

In getting our results we used the following parameters:

$$
\begin{array}{llllll}
\alpha^{-1}=137.0359895 & M_{Z}=91.1875 \mathrm{GeV} & m_{e}=0.51099907 \mathrm{MeV} & m_{\tau}=1.777 \mathrm{GeV} & m_{c}=1.5 \mathrm{GeV} & m_{t}=173.7 \mathrm{GeV} \\
& M_{W}=80.45 \mathrm{GeV} & m_{\mu}=105.658389 \mathrm{MeV} & m_{u}=m_{d}=53.8 \mathrm{MeV} & m_{s}=150 \mathrm{MeV} & m_{b}=4.7 \mathrm{GeV}
\end{array}
$$

For $\alpha_{s}$ we use a running constant $\alpha_{s}(\mu)$ with $\mu=\sqrt{s_{V V}}$. The running is evaluated at the tree-loop level within the $\overline{\mathrm{MS}}$ with 5 flavours and the normalisation $\alpha_{s}\left(M_{Z}\right)=0.1172$. We have, for instance, $\alpha_{s}\left(\sqrt{s_{W W}}=500 \mathrm{GeV}\right)=0.09432$ and $\alpha_{s}\left(\sqrt{s_{W W}}=1 \mathrm{TeV}\right)=0.08776$. We also set the CKM matrix to unity.

\subsection{Total cross section}

The effect of the radiative corrections for $M_{\text {Higgs }}=120 \mathrm{GeV}$ is shown in figure 8 for the total cross section for centre of mass energies of the $V V$ system ranging from threshold to $1 \mathrm{TeV}$. Results for $M_{\text {Higgs }}=200,300 \mathrm{GeV}$ are listed in Table 1. First of all, let us point to a common feature we found for all Higgs masses we studied. The QED corrections we extracted are relatively quite small so that there is little difference between the full EW corrections including hard radiation and what we call the "purely" EW corrections. This difference is largest, around $2 \%$ at threshold energies (around $400 \mathrm{GeV}$ ) in the case of $W^{-} W^{+} \rightarrow t \bar{t}$. Otherwise the difference is less than $1 \%$ for both $Z Z \rightarrow t \bar{t}$ and $W^{-} W^{+} \rightarrow t \bar{t}$. Therefore in the remainder of our discussion we will refer to the EW corrections having in mind the full EW. For 


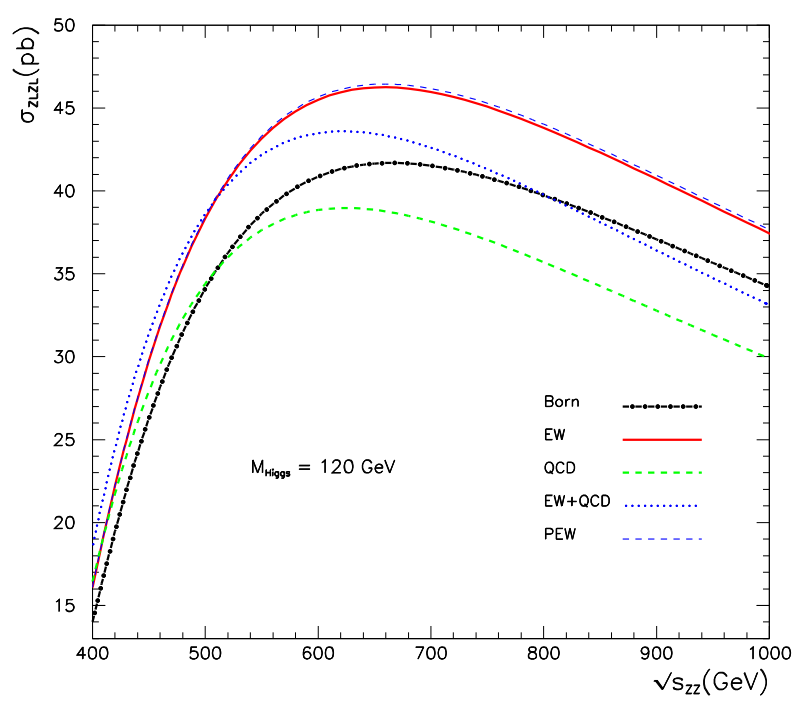

(a) $Z_{L} Z_{L} \rightarrow t \bar{t}$

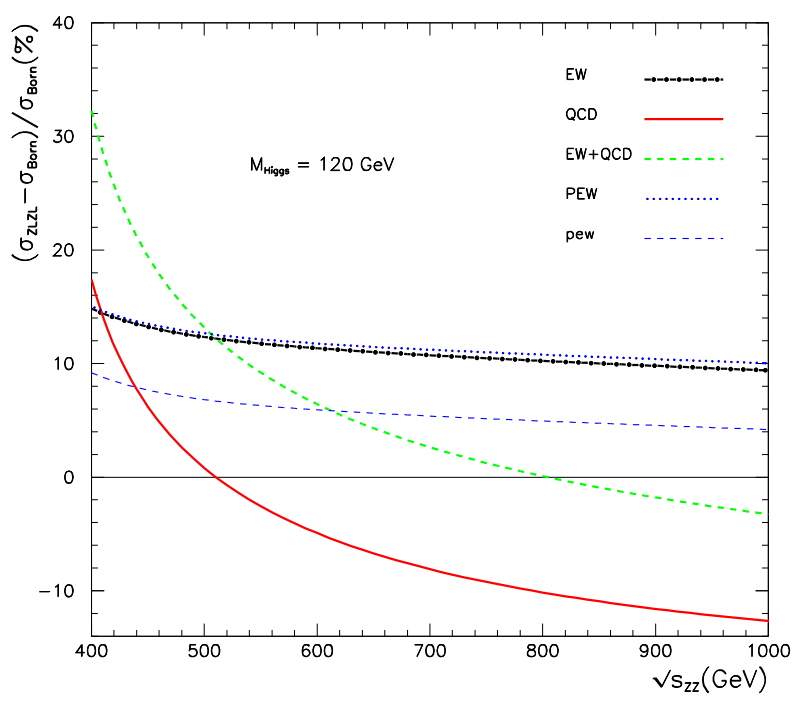

(c) $Z_{L} Z_{L} \rightarrow t \bar{t}$

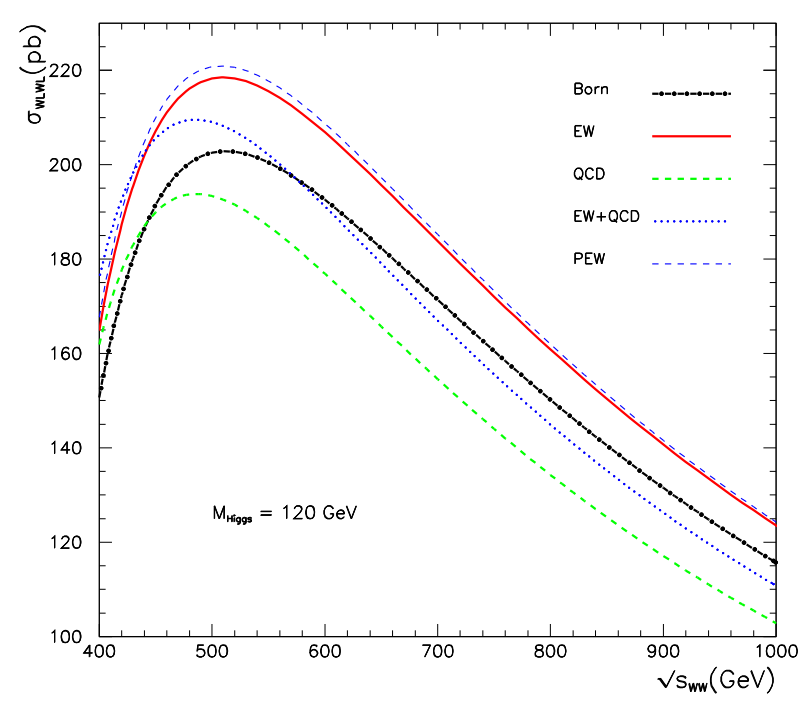

(b) $W_{L}^{-} W_{L}^{+} \rightarrow t \bar{t}$

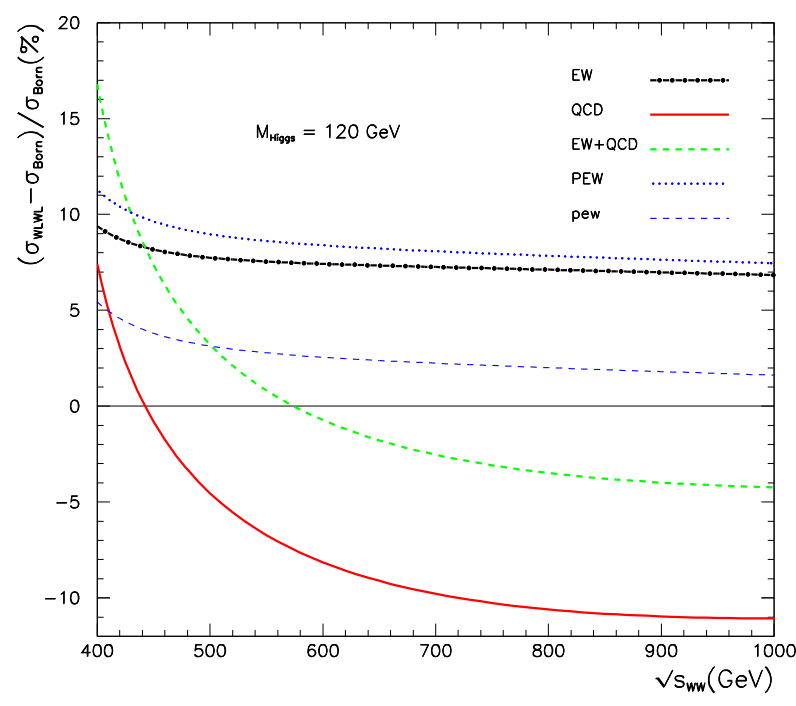

(d) $W_{L}^{-} W_{L}^{+} \rightarrow t \bar{t}$

FIG. 8: Total cross section for the Born, the NLO with full electroweak (EW), QCD, purely electroweak $(P E W)$ with the extraction of the $Q E D$ corrections and in the $G_{\mu}$ scheme(pew), see text. a) $Z_{L} Z_{L} \rightarrow t \bar{t}$ and b) $W_{L}^{-} W_{L}^{+} \rightarrow t \bar{t} ; M_{\text {Higgs }}=120 \mathrm{GeV}$. c) and d) give the percentage change. 
both $Z Z$ and $W W$, we find large positive corrections at the lower energies. As we discussed earlier, this is due, to a large extent, to the Coulomb correction. Apart from the low energy region, the corrections we find for the case of the $W W$ and $Z Z$ are within $3 \%$ of each other, be it for the EW or QCD corrections. At the threshold region though, the QCD corrections are large, especially for $Z Z$ and increase somehow as the Higgs mass is increased from $120 \mathrm{GeV}$ to $300 \mathrm{GeV}$. Past the threshold region the QCD corrections decrease quite rapidly turning negative with a correction of about $-10 \%$ at $\sqrt{s_{V V}}=1 \mathrm{TeV}$. In contrast, past the threshold region, the electroweak corrections decrease extremely slowly and are almost constant with a value of around $10 \%$. Especially at high energies, the electroweak corrections tend therefore to cancel out most of the QCD corrections. The electroweak corrections should therefore be taken into account on par with the QCD corrections. Of course, expressing the tree-level cross section in terms of $G_{\mu}$ does account for a large part of the EW corrections. In this scheme the corrections are in the range $2-5 \%$, slightly larger for $Z Z$ with $M_{\text {Higgs }}=120 \mathrm{GeV}$. However even in this scheme the corrections can be competitive with the QCD corrections as the latter become small in the energy range $\sqrt{s}=500-700$ $\mathrm{GeV}$.

\begin{tabular}{|c|c|c|c|c|c||c|c|c|c|c|}
\hline$W_{L}^{-} W_{L}^{+} \rightarrow t \bar{t}$ & \multicolumn{5}{|c||}{$M_{\text {Higgs }}=200 \mathrm{GeV}$} & \multicolumn{5}{c|}{$M_{\text {Higgs }}=300 \mathrm{GeV}$} \\
\hline$\sqrt{s_{W W}}(\mathrm{GeV})$ & $\sigma_{0}(\mathrm{pb})$ & $\delta^{E W}$ & $\delta^{P E W}$ & $\delta^{\text {pew }}$ & $\delta^{Q C D}$ & $\sigma_{0}(\mathrm{pb})$ & $\delta^{E W}$ & $\delta^{P E W}$ & $\delta^{\text {pew }}$ & $\delta^{Q C D}$ \\
\hline \hline 400 & 144.33 & 9.35 & 11.17 & 4.97 & 6.44 & 163.77 & 10.25 & 11.93 & 5.42 & 10.54 \\
\hline 500 & 196.13 & 8.23 & 9.43 & 3.23 & -4.99 & 201.23 & 9.41 & 10.52 & 4.00 & -3.97 \\
\hline 600 & 188.49 & 7.96 & 8.91 & 2.72 & -8.33 & 189.66 & 8.93 & 9.82 & 3.30 & -7.89 \\
\hline 700 & 168.84 & 7.79 & 8.61 & 2.41 & -9.87 & 169.04 & 8.67 & 9.43 & 2.92 & -9.59 \\
\hline 800 & 148.57 & 7.63 & 8.36 & 2.16 & -10.61 & 148.52 & 8.43 & 9.12 & 2.60 & -10.42 \\
\hline 900 & 130.46 & 7.48 & 8.15 & 1.95 & -10.96 & 130.36 & 8.22 & 8.84 & 2.33 & -10.81 \\
\hline 1000 & 114.95 & 7.33 & 7.95 & 1.75 & -11.07 & 114.84 & 8.01 & 8.59 & 2.08 & -10.93 \\
\hline \hline$Z_{L} Z_{L} \rightarrow t \bar{t}$ & \multicolumn{6}{|c|}{$M_{\text {Higgs }}=200 \mathrm{GeV}$} & \multicolumn{5}{|c||}{$M_{\text {Higgs }}=300 \mathrm{GeV}$} & \\
\hline$\sqrt{s_{Z Z}}(\mathrm{GeV})$ & $\sigma_{0}(\mathrm{pb})$ & $\delta^{E W}$ & $\delta^{P E W}$ & $\delta^{\text {pew }}$ & $\delta^{Q C D}$ & $\sigma_{0}(\mathrm{pb})$ & $\delta^{E W}$ & $\delta^{P E W}$ & $\delta^{\text {pew }}$ & $\delta^{Q C D}$ \\
\hline \hline 400 & 21.87 & 9.96 & 10.14 & 3.94 & 22.46 & 98.10 & 10.18 & 10.36 & 3.85 & 30.87 \\
\hline 500 & 44.24 & 10.13 & 10.46 & 4.26 & 2.93 & 96.56 & 10.19 & 10.51 & 3.99 & 7.9 \\
\hline 600 & 48.49 & 10.15 & 10.57 & 4.37 & -4.06 & 78.83 & 10.21 & 10.62 & 4.10 & -1.42 \\
\hline 700 & 46.89 & 10.04 & 10.53 & 4.33 & -7.83 & 65.39 & 10.72 & 11.20 & 4.68 & -6.55 \\
\hline 800 & 43.53 & 9.84 & 10.38 & 4.18 & -10.17 & 55.45 & 10.78 & 11.32 & 4.80 & -9.69 \\
\hline 900 & 39.81 & 9.57 & 10.16 & 3.96 & -11.77 & 47.85 & 10.61 & 11.20 & 4.68 & -11.74 \\
\hline 1000 & 36.23 & 9.27 & 9.91 & 3.71 & -12.91 & 41.87 & 10.33 & 10.96 & 4.44 & -13.14 \\
\hline
\end{tabular}

TAB. 1: The Born total cross section $\sigma_{0}$ and the relative corrections for the electroweak $\left(\delta^{E W}\right)$, "purely" electroweak ( $\left.\delta^{P E W}\right)$, the $G_{\mu}$ scheme ( $\left.\delta^{\text {pew }}\right)$ and $Q C D\left(\delta^{Q C D}\right)$.

\subsection{One loop angular distributions}

We show the corrected angular distributions for an energy not far from threshold, $\sqrt{s_{V V}}=400 \mathrm{GeV}$, and the other at the high energy of $1 \mathrm{TeV}$. The corresponding $K_{W W}^{S M}$ factors for the unpolarised case, defined to be the ratio of the one loop corrected differential cross section to the corresponding tree level one, are shown in Fig. 9, In the lower energy where the cross section is largest, see Fig. 4, the electroweak correction dominates over the QCD one. In the central region the two corrections are both positive and of very similar size. At high energy, the electroweak correction shows a very large effect in the forward region, while it is quite modest in the backward region and almost zero in the central region. The large correction in the forward region has to be put in perspective though. Indeed, in this region the differential 
cross section is 4 orders of magnitude smaller than the in the backward region. This is just an effect due to hard photon radiation that migrates some of the events from the most populated region. In the central region the electroweak correction is almost nil. The QCD correction on the other hand is negative all throughout but even more so in the backward direction. This is at the origin of the relatively large and negative correction at the level of the integrated cross section.

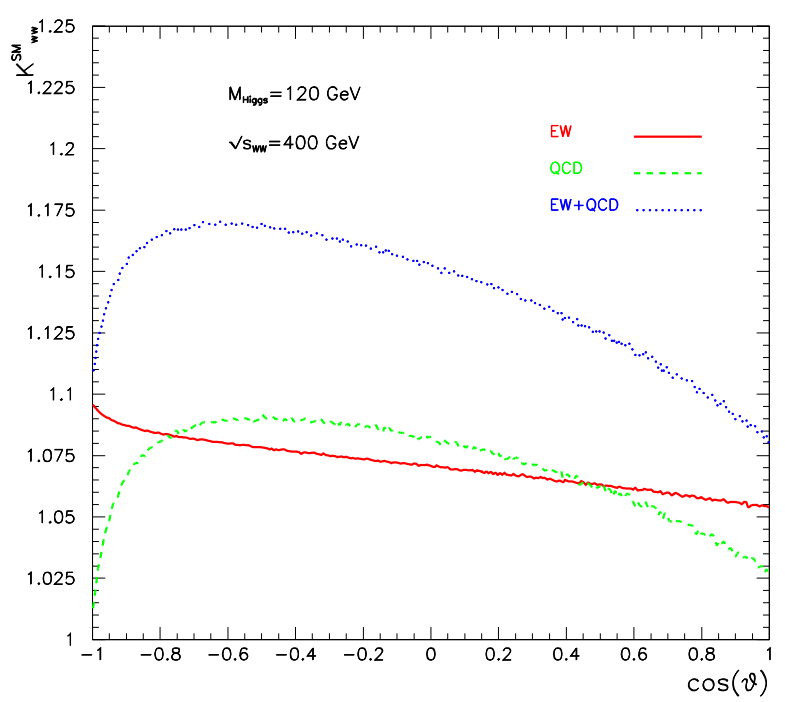

(a) $K_{W W}^{S M}$ rates for $\sqrt{s_{W W}}=400 \mathrm{GeV}$

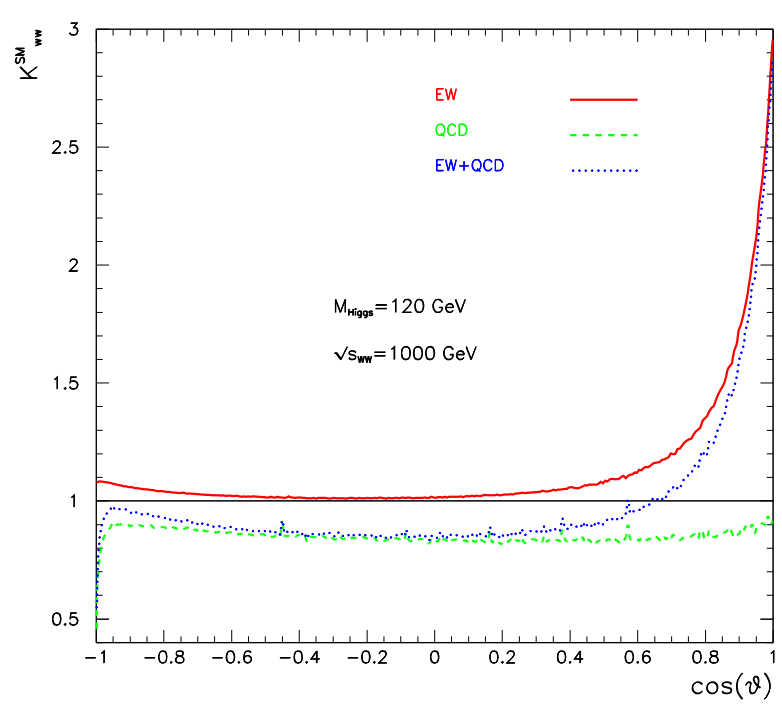

(b) $K_{W W}^{S M}$ rates for $\sqrt{s_{W W}}=1000 \mathrm{GeV}$

FIG. 9: The correction $K_{W W}^{S M}$ relative to the $\mathcal{S M}$ for the unpolarised cross section $W_{U}^{-} W_{U}^{+} \rightarrow t \bar{t}$ with $M_{\text {Higgs }}=120 \mathrm{GeV}$, (a) $\sqrt{s_{W W}}=400 \mathrm{GeV}$,(b) $\sqrt{s_{W W}}=1000 \mathrm{GeV}$. The $\mathcal{S} \mathcal{M}$ Born distributions are dispalyed in Fig. 囵.

\section{$5 \quad$ Results at $e^{+} e^{-}$level}

We now convolute the results found at the "partonic" level $V V \rightarrow t \bar{t}$ to $e^{+} e^{-} \rightarrow t \bar{t}+X$. As discussed in the previous sections we impose the kinematical cut $m_{t \bar{t}}>400 \mathrm{GeV}$ together with $p_{T}^{t, \bar{t}}>10 \mathrm{GeV}$. We have considered a centre-of mass energy for the linear collider ranging from $500 \mathrm{GeV}$ to $3 \mathrm{TeV}$. As we discussed previously, for $\sqrt{s_{e e}}<1 \mathrm{TeV}$, the EVBA approximation for the process should be taken with a grain of salt. We do this however in order to also comply with the analysis conducted in [6] and be able to compare the results for the QCD corrections we find with theirs. As in [6] we quantify the relative correction at the $e^{+} e^{-}$level as:

$$
K_{V V}=\left(\sigma_{V V}^{N L O}\right) / \sigma_{V V}^{L O} \equiv 1+\delta^{N L O} .
$$

Figure 10] shows the corrections for $M_{\text {Higgs }}=120 \mathrm{GeV}$ and $M_{\text {Higgs }}=300 \mathrm{GeV}$ as a function of the centre-of-mass energy, $\sqrt{s_{e e}}$. Our results for the $E W, P E W$ and pew corrections have a straightforward interpretation if one recalls the results obtained at the $V V$ level. Indeed, we found that the EW corrections, as the $V V$ energy is varied, is almost constant, with roughly the same correction for the $W W$ and $Z Z$ channel for the Higgs masses we studied. The correction amounts to about $10 \%$, or to about $5 \%$ in the $G \mu$ scheme. No wonder that this is roughly what we get after convolution. For the QCD part, the corrections depend on $\sqrt{s_{e e}}$. For low energies, $\sqrt{s_{e e}}=500 \mathrm{GeV}$, the QCD corrections for $Z Z$ are of order $10 \%$ for $M_{\text {Higgs }}=120 \mathrm{GeV}$ and almost twice as much for $M_{\text {Higgs }}=300 \mathrm{GeV}$. They do however drop quickly being sensibly equal to those found in $W W$, turning negative of order $-5 \%$ at $\sqrt{s_{e e}}=3 \mathrm{TeV}$. These results can again be guessed based on what we found at the $V V$ level. We completely agree with the results of [6] for the $\mathrm{QCD}$ corrections, at least for $M_{\text {Higgs }}=120 \mathrm{GeV}$ that we both consider. To 


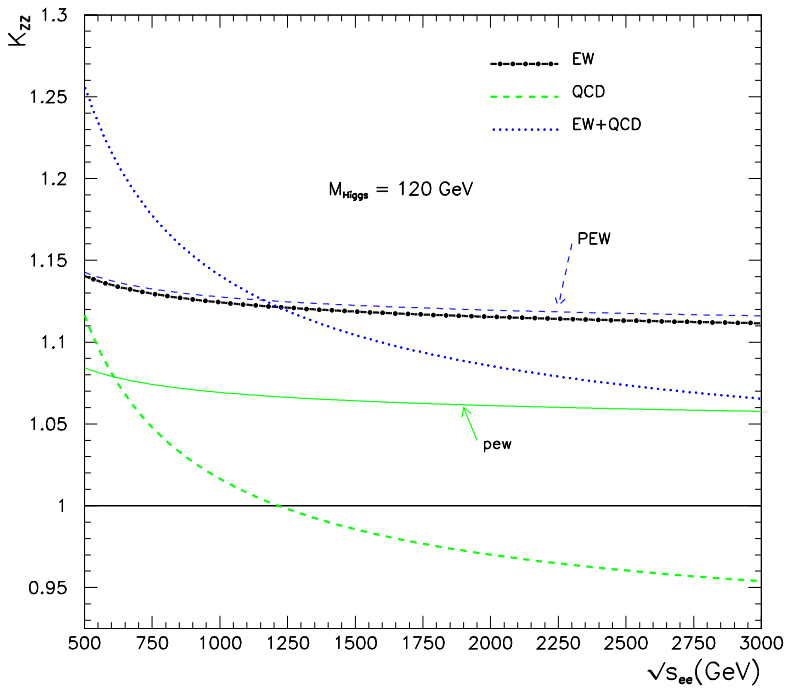

(a) $K_{Z Z}$ factor for $e^{+} e^{-} \rightarrow t \bar{t} e^{+} e^{-}$through $Z_{L} Z_{L} \rightarrow t \bar{t}$

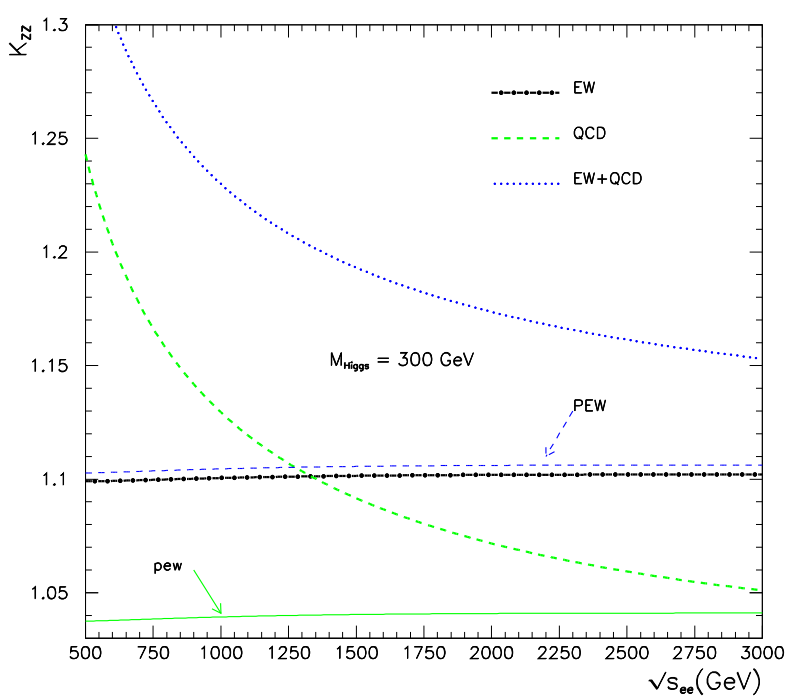

(c) $K_{Z Z}$ factor for $e^{+} e^{-} \rightarrow t \bar{t} e^{+} e^{-}$through $Z_{L} Z_{L} \rightarrow t \bar{t}$

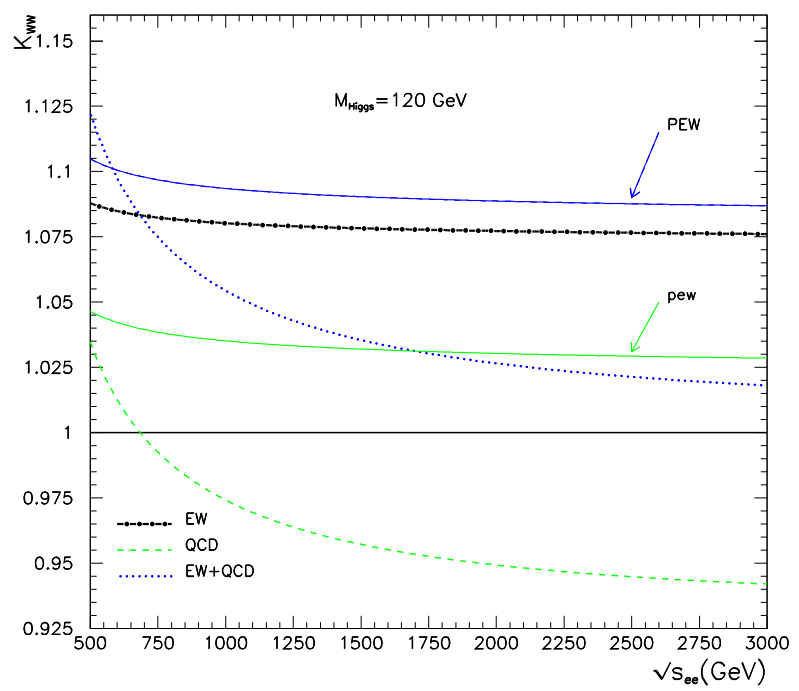

(b) $K_{W W}$ factor for $e^{+} e^{-} \rightarrow t \bar{t} \nu_{e} \overline{\nu_{e}}$ through $W_{L} W_{L} \rightarrow t \bar{t}$

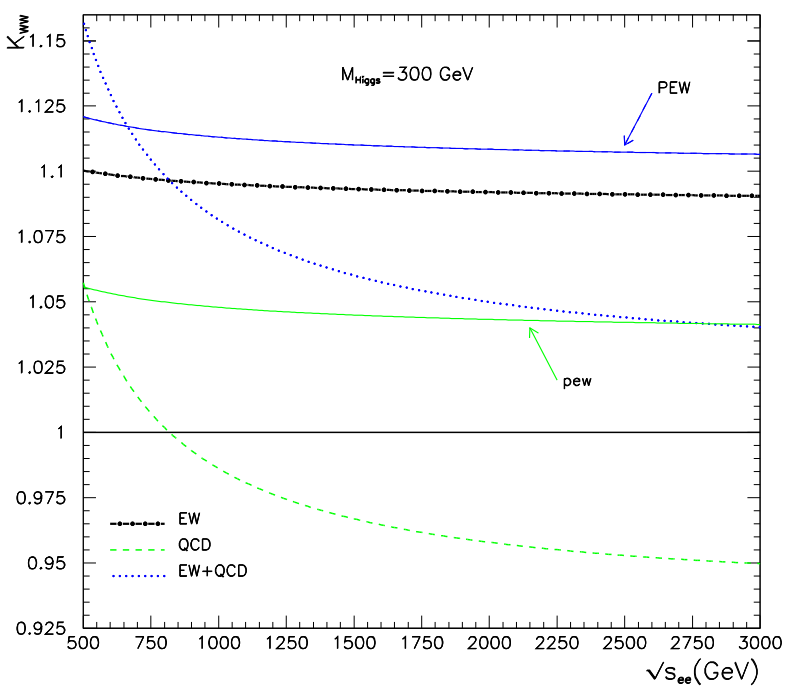

(d) $K_{W W}$ factor for $e^{+} e^{-} \rightarrow t \bar{t} \nu_{e} \overline{\nu_{e}}$ through $W_{L} W_{L} \rightarrow t \bar{t}$

FIG. 10: $K$ factor representing the loop corrections to $(a, c) e^{+} e^{-} \rightarrow t \bar{t} e^{+} e^{-}$through $Z_{L} Z_{L} \rightarrow t \bar{t}$ and $(b, d) e^{+} e^{-} \rightarrow t \bar{t} \nu \bar{\nu}$ process through $W_{L} W_{L} \rightarrow t \bar{t} .(a, b)$ are for $M_{\text {Higgs }}=120 G e V$ and $(c, d)$ for $M_{\text {Higgs }}=$ $300 \mathrm{GeV}$. 
summarise, we see that for $\sqrt{s_{e e}}=1.5 \mathrm{TeV}$ were these processes are most likely to be useful and where the EVBA can be trusted, in the $W W$ channel the electroweak corrections are of order $10 \%$ while QCD correction is of order $-5 \%$. Even translating the electroweak results in the $G \mu$ scheme, the electroweak corrections are of order $4 \%$ and practically wash out the QCD corrections.

\section{$6 \quad$ Effects of anomalous top quark interactions}

As we argued in the introduction, the processes we are studying might be a powerful tool to reveal novel interactions of the top quarks to the vector bosons, in particular their longitudinal modes. Without picking up any specific dynamical model in particular, a simple way of studying such effects is to revert to the effective Lagrangian approach. The most general lowest order operators, beyond those in the $\mathcal{S} \mathcal{M}$ have been given in 27]. We will only pick up two such operators and see their effect in $W^{-} W^{+} \rightarrow t \bar{t}$. In the unitary gauge and picking up only the $W W t \bar{t}$ couplings one can write:

$$
\mathcal{L}_{e f f}=\mathcal{L}_{e f f}^{\left(a_{1}\right)}+\mathcal{L}_{e f f}^{\left(a_{2}\right)}=\frac{a_{1}}{\Lambda} \bar{t} g^{\mu \nu} t W^{+}{ }_{\mu} W^{-}{ }_{\nu}+\frac{a_{2}}{\Lambda} \bar{t}\left(i \sigma^{\mu \nu}\right) t W^{+}{ }_{\mu} W^{-}{ }_{\nu},
$$

$a_{1}$ is a scalar interaction which contribute to the $S$-wave and $a_{2}$ is a magnetic interaction which contributes to the $P$-wave, see Appendix for the helicity amplitudes. $\Lambda$ is the scale of New Physics associated with symmetry breaking which we take in accordance with[27] to be $\Lambda=4 \pi v \sim 3.1 \mathrm{TeV}$. Reference[14 finds that $a_{1,2}$ of order 0.1 can be probed at a linear collider with $\sqrt{s_{e e}}=1.5 \mathrm{TeV}$ and an integrated luminosity of $200 \mathrm{fb}^{-1}$ in precisely the $W$ fusion process we are studying. These bounds may be improved with the use of top polarisation[27].

\subsection{Effects in $W^{-} W^{+} \rightarrow t \bar{t}$}

The $a_{i}$ we consider will be of the order 0.1 or lower as indicated by the bounds set at an $1.5 \mathrm{TeV}$ collider [14. They will contribute essentially through an interference effect with the $\mathcal{S M}$ (tree-level) amplitude contribution. Apart from their effects on the total cross section, we wish to see how they affect the distributions. This not only can tell apart the contribution of the two operators but also differentiate between the new operators and the $\mathcal{S M}$ prediction. Taking into account the $\mathcal{S} \mathcal{M}$ loop corrections one can ask whether the operators can mimick the loop corrections. For this purpose we set the value of the $a_{i}$ such that it reproduces the one-loop corrected $\mathcal{S M}$ value for the unpolarised total cross section. For example, $a_{1}^{e w}$ is the value of $a_{1}$ that reproduces the full electroweak correction,

$$
\sigma^{\text {anom } 1 e w}\left(a_{1}^{e w}\right)=\sigma_{1 l o o p}^{S M}(E W) .
$$

Likewise, $a_{i}^{q c d}$ will represent the QCD correction and $a_{i}^{e w+q c d}$ the total one-loop correction. We restrict ourselves to $M_{\mathrm{Higgs}}=120 \mathrm{GeV}$. For example, for $\sqrt{s_{W W}}=400 \mathrm{GeV}$, we find $a_{1}^{e w+q c d}=0.5$ and $a_{2}^{e w+q c d}=$ 0.15 while for $\sqrt{s_{W W}}=1000 \mathrm{GeV}$, we get $a_{1,2}^{e w}=0.02$ an order of magnitude smaller, but perhaps too optimistic from the experimental measurement, at least for $1.5 \mathrm{TeV}$ collider, but not necessarily for a $3 \mathrm{TeV}$ machine. When we look at the angular distributions, see Fig. 11, we see as expected, that in principle one can tell the different effects apart, especially for low energies. At $1 \mathrm{TeV} W W$ centre of mass, one needs to get to the central region, where unfortunately the cross section is much smaller. Nonetheless, we clearly see that one should take into account the effect of loop corrections when looking for new effects.

\subsection{Effects in $W^{-} W^{+} \rightarrow t \bar{t}$ at $e^{+} e^{-}$level: convolution}

We now convolute with the $e / W$ distribution functions for the longitudinal mode and weigh the effect of the anomalous couplings with those of the radiative corrections as a function of the $e^{+} e^{-}$centre of mass energy for $e^{+} e^{-} \rightarrow t \bar{t} \nu_{e} \overline{\nu_{e}}$. For illustration we take $a_{1}=a_{2}=0.03$. We look at the effect on, the "K-factor" $K_{W W}=\sigma^{l o o p, a_{i}} / \sigma^{\text {Born }}$. 


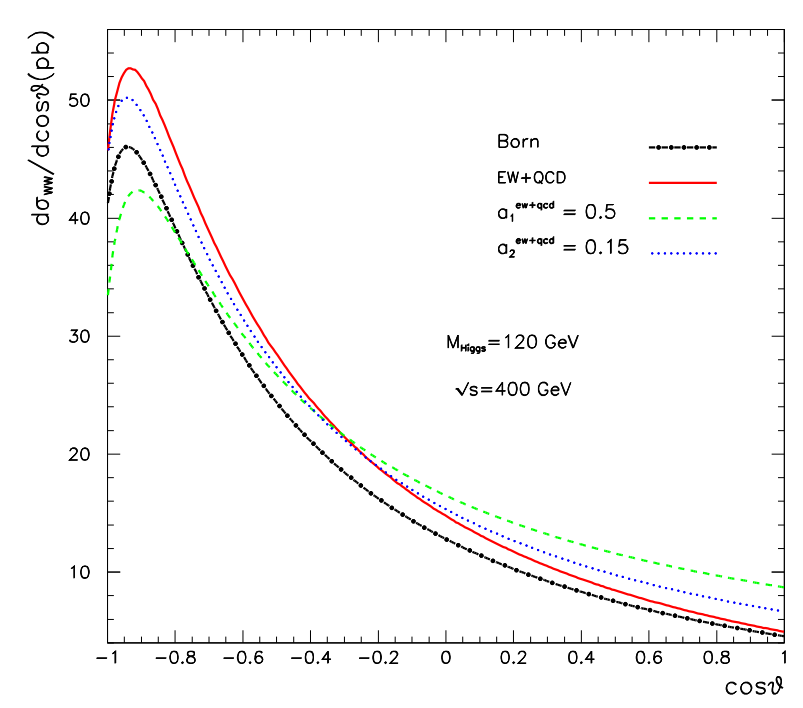

(a) Differential distributions for $\sqrt{s_{W W}}=400 \mathrm{GeV}$

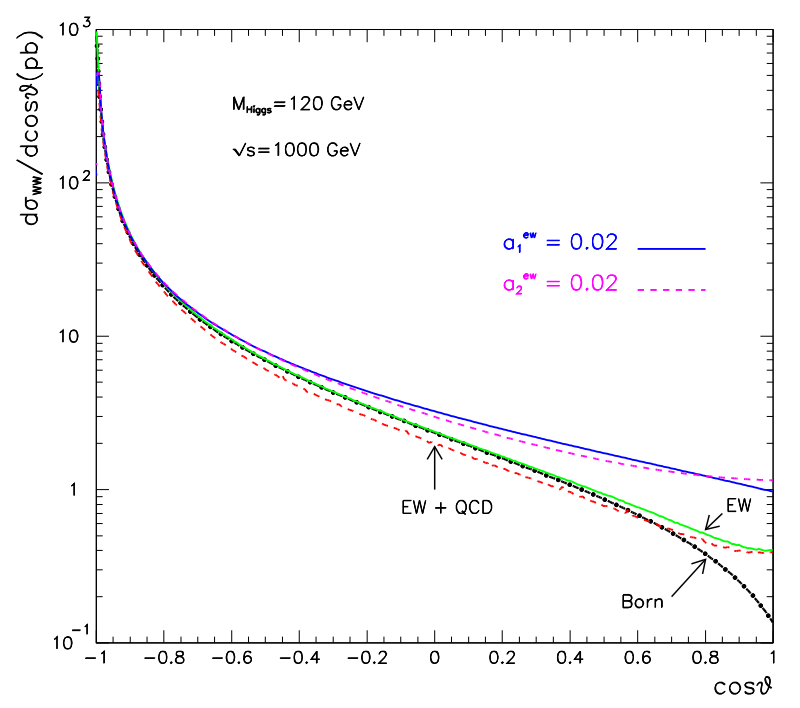

(b) Differential distributions for $\sqrt{s_{W W}}=1000 \mathrm{GeV}$

FIG. 11: Angular distributions for the SM at tree and loop level and those including the anomalous couplings that give the same total cross section as the corrected total cross section, see text. The figures are for the unpolarised cross section. $M_{\text {Higgs }}=120 \mathrm{GeV}$, for (a) $\sqrt{s_{W W}}=400 \mathrm{GeV}$, (b) $\sqrt{s_{W W}}=1000 \mathrm{GeV}$

As expected, the effect due to the anomalous couplings increases with energy. The lesson one also gets is that though one can measure a discrepancy with a tree-level calculation due to the anomalous interaction, this is not necessarily the case compared to the use of a corrected $\mathcal{S M}$ cross section. Moreover, "in real life" an anomalous contribution might be there but its effect is washed out or dramatically reduced due to the "contamination" from the QCD or the electroweak corrections, if these are not taken into account. This said, in "real life", other important issues must be taken care of such as the background and the use of a calculation that can go beyond the EVBA approximation. However, the study we have made here clearly shows that taking into account both the electroweak and QCD corrections is an important issue.
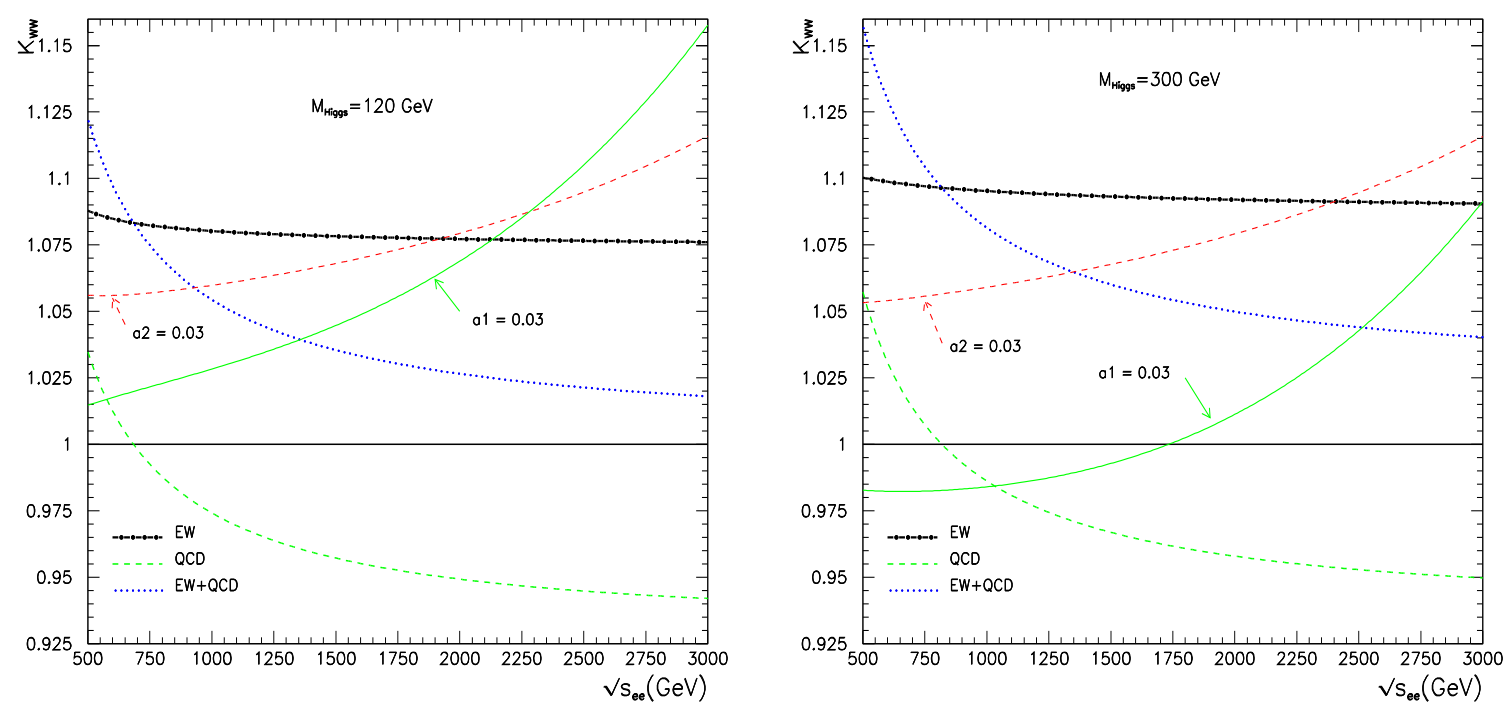

FIG. 12: $K_{W W}$ with $a_{1}=a_{2}=0.03$ for $e^{+} e^{-} \rightarrow t \bar{t} \nu_{e} \overline{\nu_{e}}$ through $W_{L} W_{L} \rightarrow t \bar{t}$ compared to the one-loop $K$-factors. 


\section{Conclusion}

There are plenty of arguments that point to the top playing a very special role in the mechanism of symmetry breaking. $W^{-} W^{+} \rightarrow t \bar{t}$ and $Z Z \rightarrow t \bar{t}$ at high energy provide a very nice testing ground for revealing features of symmetry breaking that may not be probed otherwise since they bring together the top and the Goldstone bosons. On the other hand within the $\mathcal{S M}$ and because of the large Yukawa coupling of the top, the electroweak virtual effects of the top are often large. We have performed a full electroweak correction to these processes together with the QCD corrections. The latter had been calculated recently and we agree with their findings. We find that the electroweak corrections can be large of order $10 \%$, though smaller of order $5 \%$ if we use the $G_{\mu}$ scheme. However, even in this scheme the electroweak corrections can be of order the QCD corrections and combine such that the two corrections cancel each other for a certain range of energy. The combined effect should therefore be taken into consideration when looking for New Physics effects in these processes. To illustrate the role of the radiative corrections, both QCD and electroweak, we have also considered the effect of some anomalous $W$-top interactions. Unless these are substantial enough to give deviations larger than $20 \%$ or so, one needs to take into account the radiative corrections. The latter can hide the effects or even increase it. Naturally these important processes can only occur as subprocesses at the LHC or the ILC. For the LHC, QCD top production and the QCD backgrounds are such as to prevent a study of these reactions. At the ILC, we have, like others, relied on the EVBA to study these reactions and therefore convoluted with the electron structure function describing the $W$ content of the electron. Still in this context the interplay between the electroweak and QCD corrections is important, even though for the Higgs masses we considered the convolution for $e^{+} e^{-}$centre of mass energy in the TeV range leads to the QCD and electroweak cancelling each other. Therefore taking into account the QCD corrections might not be enough to draw a meaningful conclusion in case a deviation is observed. Of course, to draw more meaningful conclusions we would need, beside a careful simulation with backgrounds, to attack the full radiative correction to $e^{+} e^{-} \rightarrow t \bar{t} \nu \bar{\nu}$, which is a formidable task. This said the gist of the electroweak corrections should be contained at the level of the $W^{-} W^{+} \rightarrow t \bar{t}$. In this study we restricted ourselves to a light enough Higgs as allowed by the precision data. It would be interesting to review the situation in the case of a very heavy Higgs or a manifestation of the $\mathcal{S M}$ without a Higgs.

\section{AKNOWLEDGMENTS}

One of us (N.B) is extremely grateful to LAPTH for its kind hospitality during the whole period this work has been conducted and to the Algerian Ministry of High Education and Sientific Research for providing a grant (BAF/20051179). N.B also wishes to thank Vincent LAFAGE for helpful discussions and for providing codes on helicity amplitude methods with massive particles.

\section{Appendix: Tree-level helicity amplitudes for $W^{-} W^{+} \rightarrow t \bar{t}$}

Let $\lambda_{1}= \pm 1$ represent the transverse modes for the incoming $W$ bosons, 0 the corresponding longitudinal mode and $\lambda_{2}= \pm 1$ the top quark polarisations. For the top quark the third component of the isospin component is $I_{3}=\frac{1}{2}$ and its charge $Q=\frac{2}{3}$. We also define $\gamma_{i}^{2}=1 /\left(1-\beta_{i}^{2}\right)=s / 4 M_{i}^{2}$ and $\pi_{i}$ the reduced propagator $\left(\pi_{i}=s \Pi_{i}\right)$, where $\sqrt{s}$ stand for the center of mass energy of the $W W$ system and

$$
\Pi_{\gamma}=\frac{1}{s}, \quad \Pi_{Z}=\frac{1}{s-M_{Z}^{2}}, \quad \Pi_{H}=\frac{1}{s-M_{\text {Higgs }}^{2}}, \quad \Pi_{b}=\frac{-4}{s\left(\beta_{t}^{2}+\beta_{W}^{2}\right)+4 m_{b}^{2}+2 s \beta_{t} \beta_{W} \cos \theta}
$$

$\theta$ is the angle between the $W^{-}$and the $t$ quark.

The tree level helicity amplitudes for the $\mathcal{S} \mathcal{M}$ are given by $\mathcal{M}_{\lambda_{1}, \lambda_{2}, \lambda_{3}, \lambda_{4}}^{S M}$ for the process $W^{-}\left(\lambda_{1}\right) W^{+}\left(\lambda_{2}\right) \rightarrow$ $t\left(\lambda_{3}\right) \bar{t}\left(\lambda_{4}\right)$

$$
\mathcal{M}_{\lambda_{1},-\lambda_{1},-\lambda_{2}, \lambda_{2}}^{S M}=-\lambda_{1} \lambda_{2} \frac{g^{2}}{8} \pi_{b} \beta_{t}\left(1-\lambda_{2} \beta_{t}\right)\left(1-\lambda_{1} \lambda_{2} \cos \theta\right) \sin \theta
$$




$$
\begin{aligned}
& \mathcal{M}_{\lambda_{1},-\lambda_{1}, \lambda_{2}, \lambda_{2}}^{S M}=\lambda_{1} \frac{g^{2}}{8} \pi_{b} \frac{\beta_{t}}{\gamma_{t}} \sin ^{2} \theta \\
& \mathcal{M}_{\lambda_{1}, \lambda_{1},-\lambda_{2}, \lambda_{2}}^{S M}=\frac{g^{2}}{4} \sin \theta\left[\frac{\pi_{b}}{2}\left(1-\lambda_{2} \beta_{t}\right)\left(\beta_{W}+\beta_{t} \cos \theta\right)+2 \pi_{Z}\left(I_{3}\left(1-\lambda_{2} \beta_{t}\right)-2 Q \frac{M_{Z}^{2}}{s} \sin ^{2} \theta_{W}\right) \beta_{W}\right] \\
& \mathcal{M}_{\lambda_{1}, \lambda_{1}, \lambda_{2}, \lambda_{2}}^{S M}=-\frac{g^{2}}{4} \frac{1}{\gamma_{t}}\left[\lambda_{1} \frac{\pi_{b}}{2}\left(\beta_{W}+\beta_{t} \cos \theta\right)\left(1+\lambda_{1} \lambda_{2} \cos \theta\right)+\lambda_{2} \pi_{H} \beta_{t}\right. \\
& \left.+2 \lambda_{2} \pi_{Z}\left(I_{3}-2 Q \frac{M_{Z}^{2}}{s} \sin ^{2} \theta_{W}\right) \beta_{W} \cos \theta\right] \\
& \mathcal{M}_{0, \lambda_{1},-\lambda_{2}, \lambda_{2}}^{S M}=\lambda_{1} \frac{g^{2}}{2 \sqrt{2}} \gamma_{W}\left(1+\lambda_{1} \lambda_{2} \cos \theta\right)\left[\frac { \pi _ { b } } { 4 } ( 1 - \lambda _ { 2 } \beta _ { t } ) \left(\beta_{W} \beta_{t}+\lambda_{1} \beta_{t}-\lambda_{2} \beta_{W}+\lambda_{1} \lambda_{2} \beta_{W}^{2}\right.\right. \\
& \left.\left.-2 \lambda_{2} \beta_{t} \cos \theta\right)-2 \lambda_{2} \pi_{Z}\left(I_{3}\left(1-\lambda_{2} \beta_{t}\right)-2 Q \frac{M_{Z}^{2}}{s} \sin ^{2} \theta_{W}\right) \beta_{W}\right] \\
& \mathcal{M}_{-\lambda_{1}, 0,-\lambda_{2}, \lambda_{2}}^{S M}=\mathcal{M}_{0, \lambda_{1},-\lambda_{2}, \lambda_{2}}^{S M} \\
& \mathcal{M}_{0, \lambda_{1}, \lambda_{2}, \lambda_{2}}^{S M}=-\sqrt{2} \frac{g^{2}}{4} \frac{\gamma_{W}}{\gamma_{t}} \sin \theta\left[\frac{\pi_{b}}{4}\left(\beta_{W} \beta_{t}+\lambda_{1} \beta_{t}+\lambda_{2} \beta_{W}-\lambda_{1} \lambda_{2} \beta_{W}^{2}+2 \lambda_{2} \beta_{t} \cos \theta\right)\right. \\
& \left.+2 \lambda_{2} \pi_{Z}\left(I_{3}-2 Q \frac{M_{Z}^{2}}{s} \sin ^{2} \theta_{W}\right) \beta_{W}\right] \\
& \mathcal{M}_{-\lambda_{1}, 0, \lambda_{2}, \lambda_{2}}^{S M}=\mathcal{M}_{0, \lambda_{1}, \lambda_{2}, \lambda_{2}}^{S M} \\
& \mathcal{M}_{0,0,-\lambda_{2}, \lambda_{2}}^{S M}=\frac{g^{2}}{4} \gamma_{W}^{2} \sin \theta\left[\frac{\pi_{b}}{2}\left(1-\lambda_{2} \beta_{t}\right)\left(2 \lambda_{2} \beta_{W} \beta_{t}-\beta_{W}\left(1-\beta_{W}^{2}\right)-2 \beta_{t} \cos \theta\right)\right. \\
& \left.-2 \pi_{Z}\left(I_{3}\left(1-\lambda_{2} \beta_{t}\right)-2 Q \frac{M_{Z}^{2}}{s} \sin ^{2} \theta_{W}\right)\left(3-\beta_{W}^{2}\right) \beta_{W}\right] \\
& \mathcal{M}_{0,0, \lambda_{2}, \lambda_{2}}^{S M}=-\lambda_{2} \frac{g^{2}}{4} \frac{\gamma_{W}^{2}}{\gamma_{t}}\left[\frac{\pi_{b}}{2}\left(\left(1+\beta_{W}^{2}\right) \beta_{t}-\left(1-\beta_{W}^{2}\right) \beta_{W} \cos \theta-2 \beta_{t} \cos ^{2} \theta\right)\right. \\
& \left.+\pi_{H}\left(1+\beta_{W}^{2}\right) \beta_{t}-2 \pi_{Z}\left(I_{3}-2 Q \frac{M_{Z}^{2}}{s} \sin ^{2} \theta_{W}\right)\left(3-\beta_{W}^{2}\right) \beta_{W} \cos \theta\right]
\end{aligned}
$$

The anomalous helicity amplitudes including the operators in Eq. 6.13 we denote by $\mathcal{N}$

$$
\begin{gathered}
\mathcal{N}_{\lambda_{1},-\lambda_{1},-\lambda_{2}, \lambda_{2}}=\mathcal{N}_{\lambda_{1},-\lambda_{1}, \lambda_{2}, \lambda_{2}}=\mathcal{N}_{\lambda_{1}, \lambda_{1},-\lambda_{2}, \lambda_{2}}=0 \\
\mathcal{N}_{\lambda_{1}, \lambda_{1}, \lambda_{2}, \lambda_{2}}=\frac{\sqrt{s}}{\Lambda} \beta_{t}\left(\lambda_{2} a_{1}+\lambda_{1} a_{2} \cos \theta\right) \\
\mathcal{N}_{0, \lambda_{1},-\lambda_{2}, \lambda_{2}}=-\lambda_{1} \lambda_{2} \frac{a_{2}}{\Lambda} \sqrt{\frac{s}{2}} \frac{\gamma_{W}}{\gamma_{t}} \beta_{W}\left(1+\lambda_{1} \lambda_{2} \cos \theta\right) \\
\mathcal{N}_{-\lambda_{1}, 0,-\lambda_{2}, \lambda_{2}}=\mathcal{N}_{0, \lambda_{1},-\lambda_{2}, \lambda_{2}}
\end{gathered}
$$




$$
\begin{gathered}
\mathcal{N}_{0, \lambda_{1}, \lambda_{2}, \lambda_{2}}=\frac{a_{2}}{\Lambda} \sqrt{\frac{s}{2}} \gamma_{W}\left(\lambda_{1} \beta_{t}-\lambda_{2} \beta_{W}\right) \sin \theta \\
\mathcal{N}_{-\lambda_{1}, 0, \lambda_{2}, \lambda_{2}}=\mathcal{N}_{0, \lambda_{1}, \lambda_{2}, \lambda_{2}} \\
\mathcal{N}_{0,0,-\lambda_{2}, \lambda_{2}}=-2 a_{2} \frac{\sqrt{s}}{\Lambda} \frac{\gamma_{W}^{2}}{\gamma_{t}} \beta_{W} \sin \theta \\
\mathcal{N}_{0,0, \lambda_{2}, \lambda_{2}}=\lambda_{2} \frac{\sqrt{s}}{\Lambda} \gamma_{W}^{2}\left(a_{1}\left(1+\beta_{W}^{2}\right) \beta_{t}+2 a_{2} \beta_{W} \cos \theta\right)
\end{gathered}
$$

These expressions completely agree with the ones found in [15]. At high energy the anomalous part is given by

$$
\mathcal{N}_{0,0,-\lambda_{2}, \lambda_{2}} \simeq-a_{2} \frac{m_{t}}{\Lambda} \frac{s}{M_{W}^{2}} \sin \theta, \quad \mathcal{N}_{0,0, \lambda_{2}, \lambda_{2}} \simeq \lambda_{2} \frac{\sqrt{s}}{2 \Lambda} \frac{s}{M_{W}^{2}}\left(a_{1}+a_{2} \cos \theta\right)
$$

This high energy behaviour agrees with the one given in 27]. The angular dependence of $a_{2}$ classifies this operator as $P$-wave, compared to $a_{1}$ which is $S$-wave[27].

\section{References}

[1] M.Beneke et al., arXiv:hep-ph/0003033;

G.Altarelli,M.L.Mangano, CERN yellow report, Geneva, 2000.

[2] J.A.Aguilar-Saavedra et al. (ECFA/DESY LC physics working group collaboration), arXiv:hep-ph/0106315;

K.Abe et al. (ACFA linear collider working group collaboration), arXiv:hep-ph/0109166].

[3] V. A. Miransky, M. Tanabashi and K. Yamawaki, Phys.Lett. B 221 (1989) 177;

W. A. Bardeen, C. T. Hill and M. Lindner, Phys. Rev. D 41 (1990)1647;

C. T. Hill, Phys.Lett. B 266 (1991) 419;

K. D. Lane and E. Eichten, Phys.Lett. B 352 (1995)382, arXiv:hep-ph/9503433;

R. S. Chivukula, E. H. Simmons and J. Terning, Phys.Lett. B 331 (1994)383, arXiv:hep-ph/9404209];

B. A. Dobrescu and C. T. Hill, Phys.Rev.Lett. 81 (1998) 2634, arXiv:hep-ph/9712319;;

R. S. Chivukula, B. A. Dobrescu, H. Georgi and C. T. Hill, Phys.Rev. D 59 (1999) 075003, arXiv:hep-ph/9809470];

H. J. He, C. T. Hill and T. M. P. Tait, Phys.Rev. D 65 (2002)055006, arXiv:hep-ph/0108041.

[4] K. Agashe, A. Belyaev, T. Krupovnickas, G. Perez and J. Virzi, arXiv:hep-ph/0612015;

B. Lillie, L. Randall and L. T. Wang, arXiv:hep-ph/0701166];

M. Guchait, F. Mahmoudi and K. Sridhar, JHEP 0705 (2007) 103, arXiv:hep-ph/0703060];

A. Djouadi, G. Moreau and R. K. Singh, arXiv:0706.4191 [hep-ph];

K. Agashe et al., arXiv:0709.0007 [hep-ph].

[5] V.M.Abazov et al. (D0 collaboration), Phys. Rev. D 75 (2007) 092001, arXiv:hep-ex/0702018].

[6] S.Godfrey,S.Zhu, Phys.Rev. D 72 (2005) 074011, arXiv:hep-ph/0511329.

[7] A.Denner, S.Dittmaier, M.Roth and L.H. Wieders, Phys.Lett. B612 (2005), arXiv:hep-ph/0502063; ibid Nucl. Phys. B724 (2005) 247, arXiv:hep-ph/0505042.

[8] F. Boudjema et al., In the Proceedings of 2005 International Linear Collider Workshop (LCWS 2005), Stanford, California, 18-22 Mar 2005, pp 0601, arXiv:hep-ph/0510184]. 
[9] R.Cahn and S.Dawson, Phys.Lett. B 136 (1984) 196; B 138(1984)464E;

S.Dawson, Nucl. Phys. B249(1985)42;

M.S.Chanowitz and M.K.Gaillard, Phys.Lett. B 142 (1984)85;

G.Kane,W.Repka and W.Rolnick, Phys.Lett. B 148 (1984) 367;

J.Lindfors, Z.Phys. C 28 (1985)427;

M.S.Chamowitz,M.K.Gaillard, Nucl.Phys., B 261 (19985)379.

[10] T. Han, D. L. Rainwater and G. Valencia, Phys.Rev.D 68 (2003) 015003, arXiv:hep-ph/0301039.

[11] LEP Collaborations: ALEPH Collaboration, DELPHI Collaboration, L3 Collaboration, OPAL Collaboration, the LEP Electroweak Working Group, arXiv:hep-ex/0612034v2].

[12] T. L. Barklow et al., In the Proceedings of 1996 DPF / DPB Summer Study on New Directions for High-Energy Physics (Snowmass 96), Snowmass, Colorado, 25 Jun - 12 Jul 1996, pp STC118, arXiv:hep-ph/9704217.

[13] J. Alcaraz and E. Ruiz Morales, Phys.Rev.Lett.86 (2001) 3726, arXiv:hep-ph/0012109.

[14] F. Larios, T. Tait and C. P. Yuan, Phys.Rev. D 57 (1998) 3106, arXiv:hep-ph/9709316.

[15] V.Lafage, Thèse de Doctorat (1996).

[16] A.Abbsabadi, W.W.Repko, D.A.Dicus and R.Vega, Phys.Rev. D 38 (1988)2770.

[17] I.Kuss,H.Spiesberger, Phys.Rev. D 53 (1996) 6078, arXiv:hep-ph/9507204.

[18] A.Pukhov et al, arXiv:hep-ph/9908288];

E.Boos et al (ComHEP collaboration), Nucl.Instrum.Meth. A534 (2004) 250, arXiv:hep-ph/0403113.

[19] F. Boudjema, A. Semenov and D. Temes, Phys.Rev. D72 (2005) 055024, arXiv:hep-ph/0507127;

F. Boudjema, A. Semenov and D. Temes, Nucl.Phys.(Proc.Suppl.) B 157 (2006) 172.

[20] T.Hann and M.Rauch, arXiv:hep-ph/0601248];

T.Hann and J.I.Illana, arXiv:hep-ph/0607049.

[21] A. Semenov. LanHEP - a package for automatic generation of Feynman rules. User's manual., arXiv:hep-ph/9608488;

A. Semenov, Nucl. Inst. Meth. A393 (1997) 293;

A. Semenov, Comp. Phys. Commun. 115 (1998) 124;

A. Semenov, arXiv:hep-ph/0208011.

[22] G.Belanger,F.Boudjema,J.Fujimoto,T.Ishikawa, T.Kaneko,K.Kato,Y.Shimizu, Phys.Rep. 430 (2006) 117, arXiv:hep-ph/0308080.

[23] G. Bélanger, F. Boudjema, J. Fujimoto, T. Ishikawa, T. Kaneko, K. Kato and Y. Shimizu, Phys. Lett. B559 (2003) 252, arXiv:hep-ph/0212261.

[24] K. Aoki, Z. Hioki, R. Kawabe, M. Konuma and T. Muta, Suppl. Prog. Theor. Phys. 73 (1982) 1.

[25] F. Boudjema and E. Chopin, Z. Phys. C73 (1996) 85, arXiv:hep-ph/9507396.

[26] G.P.Lepage, J.Comp.Phys. 27 (1978) 192;

T.Hahn, arXiv:hep-ph/0404043.

[27] F. Larios and C. P. Yuan, Phys.Rev. D 55 (1997) 7218, arXiv:hep-ph/9606397. 